\title{
Dynamical renormalization group approach to relaxation in quantum field theory
}

\author{
D. Boyanovsky ${ }^{1,2}$, 团 and H. J. de Vega ${ }^{2,1, \text { U }}$ \\ ${ }^{1}$ Department of Physics and Astronomy, University of Pittsburgh, Pittsburgh, Pennsylvania 15260, USA \\ ${ }^{2}$ LPTHE, Université Pierre et Marie Curie (Paris VI) et Denis Diderot (Paris VII), \\ Tour 16, 1er. étage, 4, Place Jussieu, 75252 Paris, Cedex 05, France
}

(Dated: October 10, 2018)

\begin{abstract}
The real time evolution and relaxation of expectation values of quantum fields and of quantum states are computed as initial value problems by implementing the dynamical renormalization group (DRG). Linear response is invoked to set up the renormalized initial value problem to study the dynamics of the expectation value of quantum fields. The perturbative solution of the equations of motion for the field expectation values of quantum fields as well as the evolution of quantum states features secular terms, namely terms that grow in time and invalidate the perturbative expansion for late times. The DRG provides a consistent framework to resum these secular terms and yields a uniform asymptotic expansion at long times. Several relevant cases are studied in detail, including those of threshold infrared divergences which appear in gauge theories at finite temperature and lead to anomalous relaxation. In these cases the DRG is shown to provide a resummation akin to Bloch-Nordsieck but directly in real time and that goes beyond the scope of Bloch-Nordsieck and Dyson resummations. The nature of the resummation program is discussed in several examples. The DRG provides a framework that is consistent, systematic and easy to implement to study the non-equilibrium relaxational dynamics directly in real time that does not rely on the concept of quasiparticle widths.
\end{abstract}

PACS numbers: 11.10.-z,05.10.Cc,11.10.Wx,05.10.-a,02.60.Nm

\section{Contents}

\section{Introduction}

II. From linear response to an initial value problem:

III. A scalar theorv

A. The Laplace transform

IV. The time evolution via Laplace transform

A. Isolated particle pole: stable particles $\quad 6$

B. Resonances:

C. Threshold singularities and Bloch-Nordsieck resummation

1. Threshold divergences

V. Dynamical renormalization group:

A. Perturbative Expansion and Secular Terms

B. Dynamical renormalization group

C. Case I: Particles and quasiparticles

D. Threshold singularities:

1. Case II: a discontinuous densitv at $\omega=\omega_{k}$.

2. Case III: a logarithmically divergent spectral density at $\omega=\omega_{k}$.

E. Summarv of dvnamical renormalization group resummation to lowest orden

VI. Specific examples in a scalar theorv:

VII. Time evolution in quantum mechanics

\footnotetext{
*Electronic address: boyan@pitt.edu

${ }^{\dagger}$ Electronic address: devega@lpthe.jussieu.fr
} 


\section{INTRODUCTION}

The study of relaxation phenomena in quantum field theory or many body systems out of equilibrium is a timely topic of multidisciplinary interest with applications ranging from ultrarelativistic heavy ion collisions, to cosmology and condensed matter physics. The program of ultrarelativistic heavy ion collisions focuses on creating a new state of matter, the Quark-Gluon plasma $[1]-[8]$ which existed about one microsecond after the Big Bang. At the ultrarelativistic heavy ion colliders RHIC at Brookhaven and the forthcoming LHC at CERN, this new state is conjectured to be formed and to exist for about $10^{-22}$ seconds. Thus probing the physics of this short lived transient state experimentally requires the theoretical understanding of non-equilibrium phenomena on unprecedented short time scales.

In cosmology the challenge of understanding non-equilibrium phenomena leading to thermalization and equilibration, phase transitions and baryogenesis $9,10,11,12$ requires a novel set of tools to study relaxation in extreme environments and on very short time scales.

In condensed matter, transport phenomena in mesoscopic systems 13 an ultrafast spectroscopy in semiconductors [14, 15] explore relaxation of non-equilibrium states on short time and distance scales.

The usual tools to study relaxation and transport phenomena is based on kinetic equations which describe the dynamics of single (quasi) particle distribution functions or the concept of the damping rate of quasiparticles which is extracted from the imaginary part of the single particle self-energy and is associated with exponential decay.

In gauge theories at high temperature it has been found that charged particles of hard momentum feature infrared singularities on their particle mass shells that lead to anomalous damping rates [16]. These infrared singularities are a consequence of the emission and absorption of transverse photons which are only dynamically screened by Landau damping.

A study of the relaxation of charged excitations directly in real time by means of a Bloch-Nordsieck resummation of the propagator 17] revealed that this anomalous damping rate actually implies non-exponential relaxation.

A novel approach, the dynamical renormalization group (DRG) was recently introduced [18, 19]. It leads to a study of relaxation and kinetics directly in real time and reveals the non-exponential relaxation of charged particles in high temperature gauge theories.

Th DRG approach to relaxation and kinetics borrows the main concepts from the study of the asymptotics in ordinary differential equations $20,21,22]$.

The dynamical renormalization group (DRG) has been recently implemented to study transport phenomena in high temperature gauge theories: in particular the electrical conductivity has been extracted in high temperature quantum electrodynamics 23]. This approach to studying relaxation and transport phenomena directly in real time does not rely on the quasiparticle concept and instead extracts the real time dynamics from a resummation program that is akin to the renormalization group in critical phenomena and deep inelastic scattering.

In this article we present the main concepts of the DRG explicitly obtaining the relaxation of expectation values in quantum field theory as well as the time evolution of quantum states.

In section II and III we begin by setting up the fully renormalized initial value problem that describes the real time evolution of the expectation value of bosonic quantum field out equilibrium in linear response. In section IV we solve the real time evolution equations in several relevant cases by Laplace transform and highlight the case in which the emission and absorption of massless quanta introduce threshold infrared divergences. These divergences render the concept of a quasiparticle decay rate ambiguous and show that a Bloch-Nordsieck resummation of these infrared divergences lead to power law relaxation at zero temperature. At finite temperature the infrared divergences are more severe and a Bloch-Nordsieck resummation of the propagator is not readily available. 
In section $\mathrm{V}$ we introduce the dynamical renormalization group resummation and compare to the results obtained by the Laplace transform solution highlighting the relevant resummations. This method allows to treat the most infrared severe cases of threshold divergences in a similar simple manner thus providing a very powerful resummation scheme.

In section VI we present a scalar theory model that features the most relevant cases: stable particles and resonances and different cases of threshold infrared divergences which are common to gauge theories.

In section VII the time evolution of states in quantum mechanics is solved in the DRG approach and a direct comparison with standard results on energy shifts and decay rates is established.

In section VIII the resummations implied by the dynamical renormalization group, and the issue of separation of time scales are discussed in detail. Section IX summarizes the conclusions.

Several appendices contain relevant mathematical details and a simple albeit illuminating example.

\section{FROM LINEAR RESPONSE TO AN INITIAL VALUE PROBLEM:}

We are interested in studying the real time evolution of expectation values of field operators. Consider a scalar field theory with an interacting Lagrangian density $\mathcal{L}[\Phi]$ the expectation value of the scalar field $\Phi$ can be obtained from linear response to an external c-number source term $J$. The appropriate formulation of real time, non-equilibrium dynamics is that of Schwinger-Keldysh 24]-26] in which a path integral along a contour in imaginary time is required to generate all of the non-equilibrium Green's functions. Along the contour the fields carry labels \pm corresponding to the forward branch $(+)$ along which the time variable takes values $-\infty \leq t \leq \infty$, and the backward branch $(-)$ along which $t$ runs back from $\infty$ to $-\infty$. The two branches describe the time evolution of a density matrix which requires the time evolution forward and backward in time (for details see refs. 24, 25, 26 ).

The non-equilibrium Lagrangian density along this contour is therefore given by [24]-[26],

$$
\mathcal{L}_{N E Q}\left[\Phi^{+}, \Phi^{-} ; J\right]=\mathcal{L}\left[\Phi^{+}\right]+J \Phi^{+}-\mathcal{L}\left[\Phi^{-}\right]-J \Phi^{-}
$$

The non-equilibrium expectation value of the scalar field in a linear response analysis is given by

$$
\left\langle\Phi^{+}(\vec{x}, t)\right\rangle=\left\langle\Phi^{-}(\vec{x}, t)\right\rangle=\phi(\vec{x}, t)=\int_{-\infty}^{+\infty} d^{3} \vec{x}^{\prime} d t^{\prime} G_{R}\left(\vec{x}-\vec{x}^{\prime}, t-t^{\prime}\right) J\left(\vec{x}^{\prime}, t^{\prime}\right),
$$

with the retarded Green's function

$$
G_{R}\left(\vec{x}-\vec{x}^{\prime}, t-t^{\prime}\right)=\left[G^{>}\left(\vec{x}-\vec{x}^{\prime}, t-t^{\prime}\right)-G^{<}\left(\vec{x}-\vec{x}^{\prime}, t-t^{\prime}\right)\right] \Theta\left(t-t^{\prime}\right)=i\left\langle\left[\Phi(\vec{x}, t), \Phi\left(\vec{x}^{\prime}, t^{\prime}\right]\right\rangle \Theta\left(t-t^{\prime}\right),\right.
$$

where the expectation value is in the full interacting theory but with vanishing source. Consider an external source term that is adiabatically switched on in time from $t \rightarrow-\infty$ and of the form,

$$
J\left(\vec{x}^{\prime}, t^{\prime}\right)=J\left(\vec{x}^{\prime}\right) e^{\epsilon t^{\prime}} \Theta\left(-t^{\prime}\right) \quad, \quad \epsilon \rightarrow 0^{+} .
$$

The retarded nature of $G_{R}\left(\vec{x}-\vec{x}^{\prime}, t-t^{\prime}\right)$ results in that,

$$
\phi(\vec{x}, t=0)=\phi_{0}(\vec{x}) \quad, \quad \dot{\phi}(\vec{x}, t<0)=0
$$

where $\phi_{0}(\vec{x})$ is determined by $J(\vec{x})$ (or vice versa), and the second line follows in the limit $\epsilon \rightarrow 0$. For a given initial value $\phi_{0}(\vec{x})$ the current $\left.J(\vec{x})\right)$ can be found from from eq. (II.2) and the vanishing of the derivative for $t<0$ is a consequence of the retarded nature of $G_{R}$.

The linear response problem with the initial conditions at $t=0$ given by eq. (II.5) can now be turned into an initial value problem for the equation of motion of the expectation value by using the (integro-) differential operator $\mathcal{O}_{(\vec{x}, t)}$ inverse of $G_{R}\left(\vec{x}-\vec{x}^{\prime}, t-t^{\prime}\right)$

$$
\mathcal{O}_{(\vec{x}, t)} \phi(\vec{x}, t)=J(\vec{x}, t) \quad, \quad \phi(\vec{x}, t=0)=\phi_{0}(\vec{x}) \quad, \quad \dot{\phi}(\vec{x}, t<0)=0,
$$

for the source term given by eq. (II.4). Within the non-equilibrium formulation, the equation of motion of the expectation value is obtained via the tadpole method [25, 26] and automatically leads to a retarded initial value problem by coupling an external source that satisfies eq.(II.4). 


\section{A SCALAR THEORY}

Although the results obtained below are generic for bosonic, fermionic or gauge theories, we will focus our analysis on the interacting bosonic theory to highlight the main ideas in a definite setting. All of the steps can be generalized to more general theories, either fermionic, bosonic and or gauge theories.

We now establish the general formulation for an arbitrary bosonic scalar field theory, deferring to specific examples in a later section. The type of theories under consideration are generally of the form

$$
\mathcal{L}=\frac{1}{2}\left(\partial_{\mu} \Phi\right)^{2}-\frac{1}{2} m_{0}^{2} \Phi^{2}+J_{0} \Phi+\mathcal{L}_{i n t}
$$

where the source coupled to the field $\Phi$ has been introduced to provide an initial value problem for its expectation value as explained in the previous section.

The fully renormalized equations of motion are obtained by introducing the usual wave function, mass and coupling renormalizations,

$$
\Phi=Z^{\frac{1}{2}} \Phi_{r} \quad, \quad J_{r}=Z^{\frac{1}{2}} J_{0} \quad, \quad m_{0}^{2} Z=m_{r}^{2} Z+\delta m^{2}
$$

with a renormalization of the interaction terms included in the interaction Lagrangian.

We now suppress the label $r$ with all quantities being renormalized, and write the Lagrangian density in terms of renormalized quantities and counterterms

$$
\begin{aligned}
\mathcal{L} & =\frac{1}{2}\left(\partial_{\mu} \Phi\right)^{2}-\frac{1}{2} m^{2} \Phi^{2}+J \Phi+\mathcal{L}_{c t}+\mathcal{L}_{i n t} \\
\mathcal{L}_{c t} & =\frac{1}{2}\left[(Z-1)\left(\partial_{\mu} \Phi\right)^{2}-m^{2} \Phi^{2}\right]-\frac{1}{2} \delta m^{2} \Phi^{2}
\end{aligned}
$$

The counterterms are adjusted in perturbation theory as usual, and the interaction Lagrangian is in terms of renormalized quantities and the counterterms associated with the interaction.

Writing,

$$
\Phi(\vec{x}, t)=\psi(\vec{x}, t)+\phi(\vec{x}, t) \quad \text { with } \quad\langle\psi(\vec{x}, t)\rangle=0 \quad ; \quad \phi(\vec{x}, t)=\langle\Phi(\vec{x}, t)\rangle,
$$

using the tadpole condition 25,26$]$

$$
\langle\psi(\vec{x}, t)\rangle=0
$$

and taking spatial Fourier transforms, we find the following equation of motion linear in the amplitude for the expectation value for $t>0$ (when the external source $J$ vanishes)

$$
\ddot{\phi}_{k}(t)+\omega_{k}^{2} \phi_{k}(t)+\int_{-\infty}^{+\infty} \Sigma_{k}^{R}\left(t-t^{\prime}\right) \phi_{k}\left(t^{\prime}\right) d t^{\prime}=0
$$

with $\omega_{k}^{2}=k^{2}+m^{2}$, and used that the adiabatic current vanishes for $t>0$ [see eq.(II.4)]. Here $\Sigma_{k}^{R}\left(t-t^{\prime}\right)$ stands for the renormalized retarded self-energy. That is, $\Sigma_{k}^{R}\left(t-t^{\prime}\right)$ is obtained from the bare one after mass and wavefunction renormalization.

As discussed in the previous section, the source is chosen so that $\phi_{k}(t=0)=\phi_{k}(0) ; \dot{\phi}_{k}(t \leq 0)=0$. $\Sigma_{k}^{R}\left(t-t^{\prime}\right)$ is the retarded self-energy which is obtained systematically in perturbation theory. The Fourier representation of the retarded self-energy is given by,

$$
\Sigma_{k}^{R}(t)=\int_{-\infty}^{+\infty} \frac{d \omega}{2 \pi} e^{-i \omega t} \Sigma_{k}(\omega)
$$

It is very useful to express the self-energy in a spectral representation. In renormalizable field theories, usually one needs to make two subtractions in the dispersion relation. However, in the scalar models considered below, the wave function renormalization is finite and one subtraction is enough (we can set $Z=1$ ). We can then write a spectral representation in the form,

$$
\Sigma_{k}(\omega+i 0)-\Sigma_{k}(0)=\omega \int_{-\infty}^{+\infty} d \omega^{\prime} \frac{\rho_{k}\left(\omega^{\prime}\right)}{\omega^{\prime}\left(\omega-\omega^{\prime}+i 0\right)}
$$


where the spectral density $\rho_{k}(\omega)$ for real bosonic fields is an odd function of $\omega$,

$$
\rho_{k}(-\omega)=-\rho_{k}(\omega)
$$

Eq.(III.7) implies that $\rho_{k}(\omega)$ is positive for positive $\omega$.

The real and imaginary parts of $\Sigma_{k}(\omega)$ are given by

$$
\operatorname{Re}\left[\Sigma_{k}(\omega)-\Sigma_{k}(0)\right]=\omega \mathcal{P} \int_{-\infty}^{+\infty} d \omega^{\prime} \frac{\rho_{k}(\omega)}{\omega^{\prime}\left(\omega-\omega^{\prime}\right)}=2 \omega^{2} \mathcal{P} \int_{0}^{+\infty} d \omega^{\prime} \frac{\rho_{k}\left(\omega^{\prime}\right)}{\omega^{\prime}\left(\omega^{2}-\omega^{\prime 2}\right)} \quad, \quad \operatorname{Im} \Sigma_{k}(\omega)=-\pi \rho_{k}(\omega)
$$

where $\mathcal{P}$ stands for the principal part.

The retarded self-energy can thus be written in a spectral representation in the form

$$
\Sigma_{k}^{R}(t)=-i \Theta(t) \int_{-\infty}^{+\infty} \rho_{k}(\omega) e^{-i \omega t} d \omega=-2 \Theta(t) \int_{0}^{+\infty} d \omega \rho_{k}(\omega) \sin \omega t
$$

and the inverse Fourier transformation,

$$
\Sigma_{k}(\omega)=\int_{0}^{+\infty} e^{i \omega t} \Sigma_{k}^{R}(t) d t \quad, \quad \operatorname{Im} \omega>0
$$

In what follows we use the usual perturbative expansions in terms of a dimensionless coupling $\lambda$ for the self-energy and the renormalization counterterms

$$
\Sigma_{k}(\omega)=\lambda \Sigma_{k}^{(1)}(\omega)+\lambda^{2} \Sigma_{k}^{(2)}(\omega)+\mathcal{O}\left(\lambda^{3}\right) \quad, \quad \rho_{k}(\omega)=\lambda \rho_{k}^{(1)}(\omega)+\lambda^{2} \rho_{k}^{(2)}(\omega)+\mathcal{O}\left(\lambda^{3}\right) .
$$

The initial value problem with the condition that $\dot{\Phi}(\vec{x}, t<0)=0$ can be solved via Laplace transform.

\section{A. The Laplace transform}

Introducing the Laplace transform of $\phi_{k}(t)$ as

$$
\varphi_{k}(s)=\int_{0}^{\infty} d t e^{-s t} \phi_{k}(t) \quad, \quad \operatorname{Re} s>0
$$

and that of the retarded self-energy,

$$
\widetilde{\Sigma}_{k}(s)=\int_{0}^{\infty} d t e^{-s t} \Sigma_{k}^{R}(t)
$$

which can be read off eq.(III.11) after the analytic continuation $i \omega=-s$,

$$
\widetilde{\Sigma}_{k}(s=-i \omega+0)=\Sigma_{k}(\omega+i 0)=\operatorname{Re} \Sigma_{k}(\omega)+i \operatorname{Im} \Sigma_{k}(\omega)
$$

where the real and imaginary parts of the self-energy are given by eqs.(III.9). Notice that $s$ approaching the imaginary axis from the right half-plane, $s=-i \omega+0$, corresponds to the causal choice $\omega+i 0$.

The spectral representation of the Laplace transform $\widetilde{\Sigma}_{k}(s)$ follows from eq. (II.7) by analytic continuation,

$$
\widetilde{\Sigma}_{k}(s)-\widetilde{\Sigma}_{k}(0)=2 s^{2} \int_{0}^{+\infty} \frac{d \omega}{\omega} \frac{\rho_{k}(\omega)}{\omega^{2}+s^{2}},
$$

where we have used the property (III.8) in the last line and $\widetilde{\Sigma}_{k}(0)=\Sigma_{k}(0)$.

Upon taking the Laplace transform, eq.(11.5) becomes the following algebraic equation (see Appendix A),

$$
\left[s^{2}+\omega_{k}^{2}+\widetilde{\Sigma}_{k}(s)\right] \varphi_{k}(s)-s \phi_{k}(0)+\frac{\phi_{k}(0)}{s}\left[\widetilde{\Sigma}_{k}(0)-\widetilde{\Sigma}_{k}(s)\right]=0 .
$$

The spectral density is consistently computed in a perturbative expansion in terms of the renormalized coupling constant. The renormalization aspects are well known and therefore we do not well into them. We just emphasize 
that the initial value problem is fully renormalized formulated in terms of the fully renormalized self-energy $\Sigma_{k}(\omega+i 0)$ for which the relation (III.15) holds in terms of the fully renormalized $\widetilde{\Sigma}_{k}(s)$.

The solution of Eq. (III.17) is therefore given by

$$
\varphi_{k}(s)=\frac{\phi_{k}(0)}{s}\left[1-\frac{\omega_{k}^{2}+\widetilde{\Sigma}_{k}(0)}{s^{2}+\omega_{k}^{2}+\widetilde{\Sigma}_{k}(s)}\right] .
$$

This expression manifestly implies that the Laplace transform method to obtain the real time evolution is equivalent to a Dyson (geometric) resummation of self-energy insertions.

The real time evolution is obtained from the inverse Laplace transform given by

$$
\phi_{k}(t)=\int_{\Gamma} \frac{d s}{2 \pi i} e^{s t} \varphi_{k}(s) .
$$

where the contour $\Gamma$ runs from $-i \infty$ to $+i \infty$ parallel to the imaginary axis in the Re $s>0$ half-plane. We note that $s=0$ is not a pole since the residue vanishes identically in eq.(III.18).

Notice that eq. (III.18) provides a non-perturbative solution for the time evolution of the expectation value of the field for a given perturbative expression for $\widetilde{\Sigma}_{k}(s)$. Eq. (III.18) is an explicit non-perturbative resummation of the Schwinger-Dyson series. For a given order in perturbation theory for the self-energy this result corresponds to a geometric (Dyson) series with the self-energy calculated at a given order in perturbation theory.

The information about the initial state is encoded in $\widetilde{\Sigma}_{k}(s)$. The self-energy is computed perturbatively with propagators containing the information about the initial state. For example, in the thermal case they depend on the initial temperature, and for non-zero density they depend on the chemical potential.

In order to compute the integral eq.(III.19) it is convenient to deform the contour to the left $s$-plane. The singularities of $\widetilde{\Sigma}_{k}(s)$ are usually along the imaginary $s$ axis, such as poles at the particle dispersion relations and production and thermal cuts.

\section{THE TIME EVOLUTION VIA LAPLACE TRANSFORM}

We now study in detail the solution of the time evolution via Laplace transform in several relevant cases.

\section{A. Isolated particle pole: stable particles}

We begin by studying the simple case in which the spectral density for $\omega>0$ has support above the position of the particle pole $\Omega_{k}\left(\sim \omega_{k}\right)$, namely

$$
\rho_{k}(\omega) \neq 0 \text { for } \Omega_{k}<\omega_{t h}<\omega \leq \infty .
$$

In this case $s^{2}+\omega_{k}^{2}+\widetilde{\Sigma}_{k}(s)$ vanishes at two points along the imaginary axis at $s= \pm i \Omega_{k}$, with $\Omega_{k}$ being the real solution of the equation

$$
\Omega_{k}^{2}-\omega_{k}^{2}-\Sigma_{k}\left(\Omega_{k}\right)=0
$$

Since $\rho_{k}\left(\Omega_{k}\right)=0$ the imaginary part of the self-energy vanishes at this point. This situation corresponds to a stable particle.

To lowest order in the coupling $\lambda$ we find,

$$
\Omega_{k}=\omega_{k}+\delta \omega_{k} \quad, \quad \delta \omega_{k}=\frac{\lambda}{2 \omega_{k}} \operatorname{Re} \Sigma_{k}^{(1)}\left(\omega_{k}\right) .
$$

The Laplace transform (III.19) is performed by wrapping the contour to the one shown in fig. \where the cuts along the imaginary axis run along $\omega_{t h} \leq|\operatorname{Im} s|<+\infty$ with $\omega_{t h}$ being the particle production threshold.

Deforming the contour $\Gamma$ to the left yields the integral as the sum of the pole contributions plus the integral over the discontinuity of the cut along the imaginary $s$-axis. This cut may be a production cut for $|\operatorname{Im} s|>\omega_{t h}$ or at finite temperature a thermal cut along $|\operatorname{Im} s|<k$ (see section VI) below. In all these cases, provided the poles are separated from the cuts, which is the case for stable particles we can write the integral in eq.(III.19) as

$$
\delta(\vec{k}, t) \equiv \frac{\phi_{k}(t)}{\phi_{k}(0)}=\mathcal{Z}_{k} \cos \left(\Omega_{k} t\right)+\left[\omega_{k}^{2}+\Sigma_{k}(0)\right] \int_{-\infty}^{+\infty} \frac{d \omega}{\omega} \frac{\rho_{k}(\omega) \cos (\omega t)}{\left[\omega^{2}-\omega_{k}^{2}-\operatorname{Re} \Sigma_{k}(\omega)\right]^{2}+\left[\operatorname{Im} \Sigma_{k}(\omega)\right]^{2}} .
$$




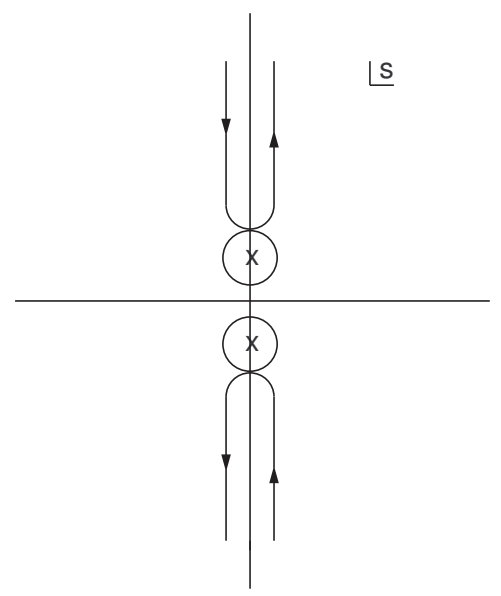

FIG. 1: Contour for the time evolution.

where

$$
\mathcal{Z}_{k}=\frac{\omega_{k}^{2}+\Sigma_{k}(0)}{\Omega_{k}^{2}\left[1+\left.\frac{1}{2 \Omega_{k}} \frac{\partial \operatorname{Re} \Sigma_{k}(\omega)}{\partial \omega}\right|_{\omega=\Omega_{k}}\right]}
$$

To lowest order in the coupling $\lambda$ we find,

$$
\mathcal{Z}_{k}=1-\lambda \int_{-\infty}^{+\infty} d \omega \frac{\rho_{k}^{(1)}(\omega)}{\omega} \mathcal{P} \frac{\omega_{k}^{2}}{\left(\omega^{2}-\omega_{k}^{2}\right)^{2}}
$$

this wave function renormalization is finite irrespective of the choice of renormalization counterterm $Z$ even in renormalizable theories where $\rho_{k}(\omega) \sim \omega^{2}$ as $\omega \rightarrow \infty$.

Evaluating the expression eq. (IV.4) at $t=0$ we arrive at the sum rule

$$
\mathcal{Z}_{k}=1-\left[\omega_{k}^{2}+\Sigma_{k}(0)\right] \int_{-\infty}^{+\infty} \frac{d \omega}{\omega} \frac{\rho_{k}(\omega)}{\left[\omega^{2}-\omega_{k}^{2}-\operatorname{Re} \Sigma_{k}(\omega)\right]^{2}+\left[\operatorname{Im} \Sigma_{k}(\omega)\right]^{2}},
$$

which is easily confirmed to lowest order in the coupling $\lambda$ by using the expression for $\Sigma_{k}$ given by eq.(III.9), the expansions (III.12) and neglecting $\Sigma_{k}^{(1)}$ in the denominator on the right hand side of eq. (IV.7).

The integral over the cut in eq.(IV.4) is usually too complicated to be computed in close form. However, we can evaluate its asymptotic behavior for late times provided we know the behavior of $\rho(k, \omega)$ near threshold.

For example, in the zero temperature case the integral over the cut in eq. (IV.4) extends over $|\omega|>\omega_{t h}$ where $\omega_{t h}$ stands for the first production threshold. Typical production cuts vanish at threshold with a power law at zero temperature,

$$
\rho_{k}(\omega) \stackrel{\omega \rightarrow \omega_{t h}}{=} A\left(\omega-\omega_{t h}\right)^{\alpha}
$$

where $A$ is a positive constant. For example for the production cut of two massive bosonic particles, the threshold behavior is determined by $\alpha=\frac{1}{2}$ and $\omega_{t h}=\sqrt{(2 m)^{2}+k^{2}}$.

We find from eq. (IV.4 for late times,

$$
\begin{aligned}
& \delta(\vec{k}, t)^{(c u t)} \equiv\left[\omega_{k}^{2}+\Sigma_{k}(0)\right] \int_{-\infty}^{+\infty} \frac{d \omega}{\omega} \frac{\rho_{k}(\omega) \cos (\omega t)}{\left[\omega^{2}-\omega_{k}^{2}-\operatorname{Re} \Sigma_{k}(\omega)\right]^{2}+\left[\operatorname{Im} \Sigma_{k}(\omega)\right]^{2}} \stackrel{t \rightarrow \infty}{=} \frac{2 A\left[\omega_{k}^{2}+\Sigma_{k}(0)\right]}{\left[\omega_{t h}^{2}-\omega_{k}^{2}-\operatorname{Re} \Sigma_{k}\left(\omega_{t h}\right)\right]^{2}} \times \\
& \times \operatorname{Re} \int_{\omega_{t h}}^{\infty} \frac{d \omega}{\omega} e^{i \omega t}\left(\omega-\omega_{t h}\right)^{\alpha} \stackrel{t \rightarrow \infty}{=} \frac{2 A\left(k^{2}+m^{2}\right) \Gamma(\alpha+1)}{\omega_{t h}\left[k^{2}+m^{2}-\omega_{t h}^{2}+\operatorname{Re} \Sigma_{k}\left(\omega_{t h}\right)\right]^{2}} \frac{1}{t^{\alpha+1}} \cos \left[\omega_{t h} t+\frac{\pi}{2}(\alpha+1)\right] .
\end{aligned}
$$

We see that the cut contribution decreases for late times with a power law. The smaller is the phase space near threshold (the larger is $\alpha$ ) the faster decreases the cut contribution for late times 27 .

Notice that eq.(IV.9) holds provided the particle pole is separated from the threshold. That is for $\omega_{t h}>\Omega_{k}$. 


\section{B. Resonances:}

In this case the imaginary part of the self energy does not vanish at $\omega_{k}$. The position of the (quasi) particle poles is determined by the equation

$$
s_{p}^{2}+\omega_{k}^{2}+\widetilde{\Sigma}_{k}\left(s_{p}\right)=0
$$

Writing $s_{p}=-i \Omega_{k}+\gamma_{k} ; \gamma_{k} \ll \Omega_{k}$ with both $\Omega_{k}$ and $\gamma_{k}$ real, and comparing real and imaginary parts we find

$$
\Omega_{k}^{2}-\omega_{k}^{2}-\operatorname{Re} \Sigma_{k}\left(\Omega_{k}\right)=0 \quad, \quad \gamma_{k}=-\operatorname{sign}\left(\gamma_{k}\right) \pi \frac{\rho_{k}\left(\Omega_{k}\right)}{2 \Omega_{k}}
$$

The first equation is the same as in the previous case eq. IV.2). Since, $\frac{\rho\left(\Omega_{k}\right)}{\Omega_{k}}>0$ unless there are instabilities, it is clear that there is no solution for the second equation. Thus there is no complex pole in the first (physical) Riemann sheet, corresponding to a resonance or quasi-particle. Since there are no poles in the first (physical) Riemann sheet in the complex $s$-plane, the Laplace transform is obtained by wrapping the contour around the imaginary axis with the result

$$
\delta(\vec{k}, t) \equiv \frac{\phi_{k}(t)}{\phi_{k}(0)}=\left[\omega_{k}^{2}+\Sigma_{k}(0)\right] \int_{-\infty}^{+\infty} \frac{d \omega}{\omega} \frac{\rho_{k}(\omega) \cos (\omega t)}{\left[\omega^{2}-\omega_{k}^{2}-\operatorname{Re} \Sigma_{k}(\omega)\right]^{2}+\left[\operatorname{Im} \Sigma_{k}(\omega)\right]^{2}}
$$

In weak coupling, the integrand of (IV.12) features a sharp peak near $\omega \simeq \Omega_{k}$, near this peak the integrand in eq. (IV.12) is of the Breit-Wigner form,

$$
\frac{\pi \rho_{k}(\omega)}{\left[\omega^{2}-\omega_{k}^{2}-\operatorname{Re} \Sigma_{k}(\omega)\right]^{2}+\left[\operatorname{Im} \Sigma_{k}(\omega)\right]^{2}} \stackrel{\omega \simeq \Omega_{k}}{=} \mathcal{Z}_{k} \frac{\Gamma_{k}}{\left(\omega-\Omega_{k}\right)^{2}+\Gamma_{k}^{2}}
$$

with $\mathcal{Z}_{k}$ given by eq.(IV.5) and

$$
\Gamma_{k} \equiv \frac{\pi \rho_{k}\left(\Omega_{k}\right)}{2 \Omega_{k}\left[1+\left.\frac{1}{2 \Omega_{k}} \frac{\partial \operatorname{Re} \Sigma_{k}(\omega)}{\partial \omega}\right|_{\omega=\Omega_{k}}\right]}
$$

To lowest order in weak coupling, $\Omega_{k}$ is given by eq. IV.3 and

$$
\Gamma_{k}=\frac{\pi \rho_{k}\left(\omega_{k}\right)}{2 \omega_{k}}+\mathcal{O}\left(\lambda^{2}\right)
$$

Since these poles are in the unphysical Riemann sheet there is no pole contribution deforming the contour $\Gamma$ in eq.(III.19). However, for small coupling the poles in the second Riemann sheet are near the cut and the sharp peak of the resonance dominates the cut contribution.

In such a case, from eq.(III.19) we find

$$
\phi_{k}(t)=\mathcal{Z}_{k} \phi_{k}(0) e^{-\Gamma_{k} t} \cos \left(\Omega_{k} t\right)+\mathcal{O}\left(\frac{1}{t} ; \lambda^{2}\right)
$$

instead of eq. IV.4. Eq. (IV.15) exhibits the Lorentz contraction of the width (dilation of lifetime) with respect to that in the rest frame $(k=0)$.

\section{Threshold singularities and Bloch-Nordsieck resummation}

Let us consider the case in which the spectral density has a threshold precisely at $\omega=\omega_{k}$, vanishing linearly at threshold. A concrete realization corresponds to a particle of mass $m \neq 0$ that emits or absorbs a massless particle (at zero temperature) with Lagrangian,

$$
L=\frac{1}{2}(\partial \Phi)^{2}+\frac{1}{2}(\partial \pi)^{2}-\frac{1}{2} M^{2} \Phi^{2}-\frac{g}{2} \Phi^{2} \pi
$$

which is a particular case of the specific examples analyzed in section (VI). 
The self-energy to one-loop takes here at zero temperature the form,

$$
\tilde{\Sigma}_{k}(s)=\frac{g^{2}}{(4 \pi)^{2}}\left(1+\frac{m^{2}}{s^{2}+k^{2}}\right) \log \left(1+\frac{s^{2}+k^{2}}{m^{2}}\right)+\mathcal{O}\left(g^{4}\right)=\lambda m^{2} \frac{s^{2}+k^{2}+m^{2}}{s^{2}+k^{2}} \log \left(\frac{s^{2}+k^{2}+m^{2}}{m^{2}}\right)+\mathcal{O}\left(\lambda^{2}\right) .
$$

where we identify the dimensionless coupling $\lambda \equiv \frac{g^{2}}{(2 \pi m)^{2}}$, and have adjusted the mass counterterm to cancel an ultraviolet logarithmic divergence.

The wave function renormalization is UV finite but features a logarithmic infrared divergence at $s^{2}=-\left(k^{2}+m^{2}\right)$. That is, $\frac{\partial \tilde{\Sigma}_{k}(s)}{\partial s}$ is divergent at $s^{2}=-\left(k^{2}+m^{2}\right)$. This infrared divergence entails that there is no longer an isolated particle pole, which now becomes the beginning of a logarithmic branch cut as a consequence of the emission and absorption of massless quanta.

The spectral density to lowest order in $\lambda$ is given by

$$
\rho_{k}^{(1)}(\omega)=m^{2} \frac{\omega^{2}-k^{2}-m^{2}}{\omega^{2}-k^{2}} \Theta\left(\omega^{2}-k^{2}-m^{2}\right) \operatorname{sign}(\omega) .
$$

We find that the inverse propagator in the Laplace transform in eq. (III.18) is given by

$$
s^{2}+k^{2}+m^{2}+\tilde{\Sigma}_{k}(s)=\left(s^{2}+k^{2}+m^{2}\right)\left[1+\lambda \frac{m^{2}}{s^{2}+k^{2}} \log \left(\frac{s^{2}+k^{2}+m^{2}}{m^{2}}\right)\right]+\mathcal{O}\left(\lambda^{2}\right) .
$$

The form of the inverse propagator given by (IV.20) indicates that in this case the logarithmic threshold behavior is associated with a wave function renormalization.

This situation is common to a wide variety of theories, in particular in Quantum Electrodynamics where the absorption and emission of massless photons introduces logarithmic threshold divergences in the electron propagator 28 which are enhanced at finite temperature 17, 19]. The logarithmic infrared divergence signals the breakdown of the perturbative expansion near the particle mass shell. The Bloch-Nordsieck method 28] provides a resummation of the leading logarithmic contributions and is equivalent to a renormalization group improvement of the propagator and the self energy. It leads [28] to the following resumed form of the inverse propagator near the mass shell $s^{2}+k^{2}+m^{2} \rightarrow 0$,

$$
s^{2}+k^{2}+m^{2}+\widetilde{\Sigma}_{k}(s) \rightarrow\left(s^{2}+k^{2}+m^{2}\right)^{1-\lambda} .
$$

The inverse Laplace transform with this resummed propagator [see eq. III.19 can be done by deforming the contour leading to the following integral representation,

$$
\phi_{k}(t)=\phi_{k}(0) \frac{2 \sin \pi \lambda}{\pi} \int_{\omega_{k}}^{\infty} \frac{d \omega}{\omega} \frac{\cos \omega t}{\left(\frac{\omega^{2}}{\omega_{k}^{2}}-1\right)^{1-\lambda}}
$$

While the integral can be computed exactly in terms of Bessel and Struve functions [18], the asymptotic long time limit is dominated by the threshold behavior at the lower bound of integration. We find for $m t \gg 1$,

$$
\phi_{k}(t) \stackrel{m t \gg 1}{=} \phi_{k}(0)\left(\frac{2}{\omega_{k} t}\right)^{\lambda} \frac{\cos \left(\omega_{k} t+\frac{\pi \lambda}{2}\right)}{\Gamma(1-\lambda)}\left[1+\mathcal{O}\left(\frac{1}{\omega_{k} t}\right)\right] .
$$

Thus a Bloch-Nordsieck or renormalization group resummation of threshold infrared divergences leads to an asymptotic long time relaxation featuring an anomalous dimension.

A perturbative expansion of eq. IV.23 in powers of the coupling $\lambda$ leads to

$$
\phi_{k}(t)=\phi_{k}(0) \cos \left(\omega_{k} t\right)\left[1-\lambda \ln \omega_{k} t+\mathcal{O}\left(\lambda^{2}, \frac{1}{\omega_{k} t}\right)\right],
$$

the logarithmic secular term signals the breakdown of the perturbative expansion. The Bloch-Nordsieck resummation of the infrared threshold divergences in the propagator leads to relaxation in terms of a power law with an anomalous dimension $\lambda$. We will see below that the logarithmic secular term in eq. (IV.24) will emerge in the perturbative expansion in real time and that the dynamical renormalization group will provide a resummation of these secular terms leading to the same result as obtained from the Bloch-Nordsieck resummation, given by eq.(IV.23) above.

Anomalous power law relaxation has been found in condensed matter physics in models of mesoscopic transport 13 and in the X-ray absorption edge singularities in metals [29] where the spectral density also features logarithmic singularities at threshold [29], as well as in the relaxation of fermionic excitations in cold dense QCD [30]. 


\section{Threshold divergences}

Consider the case in which the spectral density near $\omega=\omega_{k}$ is of the form

$$
\rho_{k}^{(1)}(\omega)=m^{2}\left[\frac{\omega^{2}-k^{2}-m^{2}}{\omega^{2}-k^{2}}+b_{k}\right] \Theta\left(\omega-\omega_{k}\right) \operatorname{sign}(\omega),
$$

with $b_{k} \neq 0$. This corresponds to the nonzero temperature case in the model (IV.17).

Following the steps outlined above, we now find that near the particle mass shell the inverse propagator is now given by,

$s^{2}+k^{2}+m^{2}+\widetilde{\Sigma}_{k}(s) \stackrel{\omega \rightarrow \omega_{k}}{=}\left(s^{2}+k^{2}+m^{2}\right)\left[1+\lambda \frac{m^{2}}{s^{2}+k^{2}} \ln \left(\frac{s^{2}+k^{2}+m^{2}}{m^{2}}\right)\right]-\lambda m^{2} b_{k} \ln \left[\frac{s^{2}+k^{2}+m^{2}}{m^{2}}\right]+\mathcal{O}\left(\lambda^{2}\right)$

and we have chosen $Z=1$. Whereas the logarithmic divergence near the particle mass shell (from the zero temperature contribution) can be interpreted in terms of a wave function renormalization and exponentiated via the Bloch-Nordsieck or renormalization group resummation, the nonzero temperature contribution $b_{k}$ leads to a stronger divergence, since not only the derivative of the inverse propagator but now the inverse propagator itself diverges at threshold. This strong divergence cannot be resummed either by the renormalization group or Bloch-Nordsieck resummation.

As we shall see below, the dynamical renormalization group in real time provides in this case a resummation that extracts the asymptotic long time behavior.

\section{DYNAMICAL RENORMALIZATION GROUP:}

We obtain here the perturbative expansion of the field expectation value and its late time behavior exhibiting secular terms. Then, we resum the secular terms using the DRG.

\section{A. Perturbative Expansion and Secular Terms}

Expanding the Laplace transform eq.([I.18) in powers of $\lambda$ yields,

$$
\varphi_{k}(s)=\phi_{k}(0)\left\{\frac{s}{s^{2}+\omega_{k}^{2}}+\lambda\left[\frac{\widetilde{\Sigma}_{k}^{(1)}(s)-\widetilde{\Sigma}_{k}^{(1)}(0)}{s\left(s^{2}+\omega_{k}^{2}\right)}-\frac{s \widetilde{\Sigma}_{k}^{(1)}(s)}{\left(s^{2}+\omega_{k}^{2}\right)^{2}}\right]+\mathcal{O}\left(\lambda^{2}\right)\right\} .
$$

The real time evolution follows by inverse Laplace transform order by order in perturbation theory. It is convenient first to express the self-energy as a dispersion relation [see eq.(III.16] yielding,

$$
\varphi_{k}(s)=\phi_{k}(0)\left\{\frac{s}{s^{2}+\omega_{k}^{2}}-\lambda\left[\frac{s \widetilde{\Sigma}_{k}^{(1)}(0)}{\left(s^{2}+\omega_{k}^{2}\right)^{2}}-2 \omega_{k}^{2} \int_{0}^{\infty} \frac{d \omega}{\omega} \rho_{k}^{(1)}(\omega) \frac{s}{\left(s^{2}+\omega_{k}^{2}\right)^{2}\left(s^{2}+\omega^{2}\right)}\right]+\mathcal{O}\left(\lambda^{2}\right)\right\} .
$$

The inverse Laplace transform of eq. $(\overline{V .2}$ is easily obtained comparing with the elementary integrals,

$$
\begin{aligned}
& \frac{s}{s^{2}+\omega_{k}^{2}}=\int_{0}^{\infty} d t e^{-s t} \cos \omega_{k} t \\
& \frac{s}{\left(s^{2}+\omega_{k}^{2}\right)^{2}}=\frac{1}{2 \omega_{k}} \int_{0}^{\infty} t d t e^{-s t} \sin \omega_{k} t \\
& \frac{s}{\left(s^{2}+\omega_{k}^{2}\right)^{2}\left(s^{2}+\omega^{2}\right)}=\frac{1}{\left(\omega^{2}-\omega_{k}^{2}\right)^{2}} \int_{0}^{\infty} d t e^{-s t}\left[\cos \omega t-\cos \omega_{k} t+\frac{\omega^{2}-\omega_{k}^{2}}{2 \omega_{k}} t \sin \omega_{k} t\right] .
\end{aligned}
$$

We find comparing eqs.(․2) and (․3) and then using eq.(III.16),

$$
\phi_{k}(t)=\phi_{k}(0)\left\{\cos \omega_{k} t+\lambda\left[2 \omega_{k}^{2} \mathcal{P} \int_{0}^{\infty} \frac{d \omega}{\omega} \frac{\rho_{k}^{(1)}(\omega)}{\left(\omega^{2}-\omega_{k}^{2}\right)^{2}}\left(\cos \omega t-\cos \omega_{k} t\right)-\frac{t}{2 \omega_{k}} \sin \omega_{k} t \operatorname{Re} \Sigma_{k}^{(1)}\left(\omega_{k}\right)\right]+\mathcal{O}\left(\lambda^{2}\right)\right\} .
$$


The second term of order $\lambda$ turns to be the frequency shift $\delta \omega_{k}$ given by eq.(IV.3.

To first order in $\lambda$ we finally have,

$$
\phi_{k}(t)=\phi_{k}(0)\left\{\cos \omega_{k} t-t \delta \omega_{k} \sin \omega_{k} t+2 \lambda \omega_{k}^{2} \mathcal{P} \int_{0}^{\infty} \frac{d \omega}{\omega} \frac{\rho_{k}^{(1)}(\omega)}{\left(\omega^{2}-\omega_{k}^{2}\right)^{2}}\left(\cos \omega t-\cos \omega_{k} t\right)+\mathcal{O}\left(\lambda^{2}\right)\right\} .
$$

The second term is a secular term: it grows as $t$. Furthermore, the frequency integral in the third term may also feature secular terms at long times. Its $t$ dependence for late times is determined by the behavior of $\rho_{k}^{(1)}(\omega)$ for $\omega \rightarrow \omega_{k}$ as we analyze below in several relevant cases.

\section{B. Dynamical renormalization group}

In perturbation theory we find that the solution of the evolution equation has in general the asymptotic form,

$$
\phi_{k}(t)=\frac{1}{2} \phi_{k}(0) e^{i \omega_{k} t}\left[1+\lambda \mathcal{S}_{k}^{(1)}(t)+\lambda^{2} \mathcal{S}_{k}^{(2)}(t)+\text { non secular }+\mathcal{O}\left(\lambda^{3}\right)\right]+\text { c.c. }
$$

with $\mathcal{S}_{k}^{(1,2)}(t)$ containing only secular terms, namely terms that grow in time.

The dynamical renormalization group absorbs the secular terms into a renormalization of the complex amplitude at an arbitrary time scale $\tau$. This is achieved by introducing a multiplicative renormalization in the following manner,

$$
\phi_{k}(0)=\phi_{k}(\tau) R_{k}(\tau)
$$

with $R_{k}(\tau)$ found systematically in an expansion in the coupling $\lambda$, namely

$$
R_{k}(\tau)=1+\lambda r_{k}^{(1)}(\tau)+\lambda^{2} r_{k}^{(2)}(\tau)+\mathcal{O}\left(\lambda^{3}\right)
$$

The coefficients $r_{k}^{(i)}(\tau)$, which play the rôle of counterterms, are chosen to cancel the secular terms at the time $t=\tau$ order by order in the perturbative expansion. To lowest order in $\lambda$ we find,

$$
r_{k}^{(1)}(\tau)=-\mathcal{S}_{k}^{(1)}(\tau)
$$

The solution renormalized in this way at a scale $\tau$ is to lowest order in $\lambda$ given by

$$
\phi_{k}(t)=\phi_{k}(\tau)\left\{1+\lambda\left[\mathcal{S}_{k}^{(1)}(t)-\mathcal{S}_{k}^{(1)}(\tau)\right]+\text { non secular }+\mathcal{O}\left(\lambda^{2}\right)\right\}+\text { c.c. }
$$

Since $\tau$ is an arbitrary scale, the solution must not depend on it. We therefore request

$$
\frac{\partial}{\partial \tau} \phi_{k}(t)=0
$$

This is the dynamical renormalization group equation.

Inserting eq. (V.10) into eq.(V.11) to lowest order in the coupling, the dynamical renormalization group equation becomes,

$$
\frac{\partial}{\partial \tau} \ln \left[\phi_{k}(\tau)\right]=\mathcal{S}_{k}^{(1)}(\tau)
$$

with solution

$$
\phi_{k}(\tau)=\phi_{k}\left(\tau_{0}\right) e^{\mathcal{S}_{k}^{(1)}(\tau)-\mathcal{S}_{k}^{(1)}\left(\tau_{0}\right)}+\text { c.c. }
$$

Since $\phi_{k}(t)$ is independent of $\tau$ thanks to the DRG eq. (V.11), we can choose the arbitrary scale $\tau$ to coincide with $t$. This yields,

$$
\phi_{k}(t) \stackrel{t \rightarrow \infty}{=} \phi_{k}(0) e^{\mathcal{S}_{k}^{(1)}(t)}\left[1+\mathcal{O}\left(\lambda^{2}, \frac{1}{t}\right)\right]+\text { c.c. }
$$

This is the long time asymptotic behavior given by dynamical renormalization group. The overall multiplicative normalization can be adjusted up to $\mathcal{O}\left(\lambda^{2}\right)$ using eq.(V.6). The next sections extract the leading secular term $\mathcal{S}_{k}^{(1)}(t)$ in several cases. 


\section{Case I: Particles and quasiparticles}

We consider first the case where the frequency $\omega_{k}$ is away from the multiparticle thresholds, namely

$$
\omega_{k} \neq \omega_{t h} ; \rho_{k}(\omega>0) \neq 0 \text { for } \omega_{t h}<\omega<+\infty
$$

That is, the spectral density is continuous, finite and non-zero at $\omega=\omega_{k}$. This case treats on equal footing the situations corresponding to a stable particle $\omega_{k}<\omega_{t h}$ and that of a quasiparticle $\omega_{k}>\omega_{t h}$.

The long time limit $t \rightarrow \infty$ can be extracted from eq. (V.5) by using formulae (B.1) in appendix B We find the asymptotic long time behavior to be given in this case by

$$
\begin{aligned}
\phi_{k}(t) & =\frac{\phi_{k}(0)}{2} e^{i \omega_{k} t}\left\{1+\lambda\left[t \frac{i \operatorname{Re} \Sigma_{k}^{(1)}\left(\omega_{k}\right)}{2 \omega_{k}}-t \frac{\pi \rho_{k}^{(1)}\left(\omega_{k}\right)}{2 \omega_{k}}+\right.\right. \\
& \left.\left.-\mathcal{P} \int d \omega \frac{\rho_{k}^{(1)}(\omega)}{\omega} \frac{\omega_{k}^{2}}{\left(\omega^{2}-\omega_{k}^{2}\right)^{2}}+\mathcal{O}\left(\frac{1}{t}\right)\right]+\mathcal{O}\left(\lambda^{2}\right)\right\}+ \text { c.c. } .
\end{aligned}
$$

The perturbative solution displays secular terms that in this case grow linearly in time. The secular terms invalidate the perturbative expansion for late times $t \gg \frac{1}{m \lambda}$.

Clearly, a resummation scheme must be invoked to resum the perturbative expansion.

In the notation of eq. (V.6) we have,

$$
\mathcal{S}_{k}^{(1)}(t)=\frac{t}{2 \omega_{k}}\left[i \operatorname{Re} \Sigma_{k}^{(1)}\left(\omega_{k}\right)-\pi \rho_{k}^{(1)}\left(\omega_{k}\right)\right] .
$$

Therefore, eq. (V.14 for the DRG solution in the long time limit takes here the form,

$$
\begin{aligned}
\phi_{k}(t) & =\mathcal{Z}_{k} \phi_{k}(0) \cos \left(\left[\omega_{k}+\delta \omega_{k}\right] t\right) e^{-\Gamma_{k} t}+\mathcal{O}\left(\frac{1}{t}, \lambda^{2}\right), \\
\delta \omega_{k} & =\lambda \frac{\operatorname{Re} \Sigma_{k}^{(1)}\left(\omega_{k}\right)}{2 \omega_{k}} ; \Gamma_{k}=\lambda \frac{\pi \rho_{k}^{(1)}\left(\omega_{k}\right)}{2 \omega_{k}}
\end{aligned}
$$

This can also be written as,

$$
\phi_{k}(t)=\mathcal{Z}_{k} \phi_{k}(0) \cos \left(\Omega_{k} t\right) e^{-\Gamma_{k} t}+\mathcal{O}\left(\frac{1}{t}, \lambda^{2}\right),
$$

with $\Omega_{k}, \mathcal{Z}_{k}$ and $\Gamma_{k}$ given by equations (IV.3), (IV.6) and (IV.15) respectively.

Comparing this DRG solution with that obtained from the Laplace transform, given by eq.(IV.16), we see that in this case the dynamical renormalization group provides a resummation akin to that of the Dyson series.

If $\rho_{k}\left(\omega_{k}\right)=0$, namely the (stable) particle pole is below the multiparticle threshold, then $\Gamma_{k}=0$ and the solution describes the asymptotic time evolution of a stable particle. On the other hand if $\omega_{k}$ is above the multiparticle threshold, then the pole moves off the physical sheet into the second Riemann sheet becoming a quasiparticle pole. This case describes a decaying particle or resonance.

\section{Threshold singularities:}

We consider in this subsection the case where the spectral density is discontinuous at $\omega=\omega_{k}$ (case II) or it exhibits a logarithmic singularity at this point (case III).

\section{Case II: a discontinuous density at $\omega=\omega_{k}$.}

Let us consider the case in which the spectral density around $\omega=\omega_{k}$ is given by equation (IV.25).

This case reflects a discontinuity at threshold since $\rho_{k}^{(1)}\left(\omega=\omega_{k}-0\right)=0 ; \rho_{k}^{(1)}\left(\omega=\omega_{k}+0\right)=A_{k}$ b and near threshold $\omega \sim \omega_{k}$ the spectral density is approximated by

$$
\rho_{k}^{(1)}(\omega)=A_{k}\left[\omega-\omega_{k}+b_{k}\right]\left[1+\mathcal{O}\left(\omega-\omega_{k}\right)\right] \Theta\left(\omega-\omega_{k}\right), A_{k}, b_{k}>0
$$


We can now extract the asymptotic long time behavior for $\phi(t)$ given by equation (V.5) by using the results given by eqs. (B.2), (B.6) and (B.7) in appendix B. We find,

$$
\begin{aligned}
& \phi_{k}(t) \stackrel{t \rightarrow \infty}{=} \frac{\phi_{k}(0)}{2} e^{i \omega_{k} t}\left\{1+i t\left[\delta \omega_{k}-\lambda \frac{A_{k} b_{k}}{2 \omega_{k}} \log \left(\omega_{k} t e^{\gamma-1}\right)\right]\right. \\
& \left.-\lambda \frac{A_{k}}{2 \omega_{k}}\left[\frac{\pi}{2} t b_{k}+\left(1-\frac{2 b_{k}}{\omega_{k}}\right) \log \left(\omega_{k} t e^{\gamma-1}\right)\right]+\mathcal{O}\left(t^{0}\right)\right\}+c . c+\mathcal{O}\left(\lambda^{2}\right),
\end{aligned}
$$

With the convention $\Theta(0) \equiv \frac{1}{2}$ we see that the real linear secular term is given by $\Gamma_{k}^{(1)} t \quad$ with $\Gamma_{k}^{(1)}=$ $-\operatorname{Im} \Sigma_{k}^{(1)}\left(\omega_{k}\right) / 2 \omega_{k}=\frac{\pi A_{k} b_{k}}{4 \omega_{k}}$

In particular, for $b_{k}=0$ we identify the secular term $-\lambda \ln \left[\omega_{k} t\right]$ which is manifest in the perturbative expansion eq. (IV.24) of the Bloch-Nordsieck resummed result given by eq.(IV.23).

Up to $\mathcal{O}(\lambda)$ we now find eq. V.21 given by eq.

$$
\mathcal{S}_{k}^{(1)}(t)=i t\left[\delta \omega_{k}-\lambda \frac{A_{k} b_{k}}{2 \omega_{k}} \log \left(\omega_{k} t e^{\gamma-1}\right)\right]-\lambda \frac{A_{k}}{2 \omega_{k}}\left[\frac{\pi}{2} t b_{k}+\left(1-\frac{2 b_{k}}{\omega_{k}}\right) \log \left(\omega_{k} t e^{\gamma-1}\right)\right] .
$$

The DRG resummation eq.(V.14) takes here the form,

$$
\phi_{k}(t) \stackrel{t \rightarrow \infty}{=} \phi_{k}(0) \cos \left[\Xi_{k}(t) t\right] e^{-\Gamma_{k} t}\left[\frac{t_{0}}{t}\right]^{\nu_{k}}+\mathcal{O}\left(\frac{1}{t} ; \lambda^{2}\right)
$$

$\Xi_{k}(t), \Gamma_{k}$ and $\nu_{k}$ are given by

$$
\begin{aligned}
\Xi_{k}(t) & =\omega_{k}+\delta \omega_{k}+\frac{\lambda A_{k} b_{k}}{2 \omega_{k}} \ln \left(\omega_{k} t e^{\gamma-1}\right) \\
\nu_{k} & =\frac{\lambda A_{k}}{2 \omega_{k}}\left(1-\frac{2 b_{k}}{\omega_{k}}\right), \quad \Gamma_{k}=\frac{\pi \lambda A_{k} b_{k}}{4 \omega_{k}}
\end{aligned}
$$

Thus, we see that the logarithmic mass shell singularity proportional to $b_{k}$ in the perturbative expansion of the propagator, eq.(IV.26), is manifest in the real-time perturbative expansion given by eq.(V.22) as a secular term of the form $t \ln [t]$. The dynamical renormalization group resums this secular term into a logarithmic phase in the field expectation value eq. (V.23).

Notice from eqs. (V.20) and (IV.19) that $A_{k}=2 \omega_{k}$ and $b_{k}=0$ for the model (IV.17). Hence, we see in eq. (V.24) that $\nu_{k}=\lambda$ in this case. Therefore, eqs. (IV.23) and (V.23) fully agree. In addition, eq. (IV.23) implies $t_{0}=2 e^{-\gamma / \omega_{k}}$ for the reference time scale.

Thus, an important conclusion resulting from the comparison between the time evolution obtained from the BlochNordsieck resummed propagator given by eq.(IV.23) for $b_{k}=0$ and the dynamical renormalization group resummation in this case, is that the DRG resummation is a Bloch-Nordsieck resummation in real time.

Moreover, while a Bloch-Nordsieck resummation of the Euclidean propagator for $b_{k} \neq 0$ given by eq. (IV.26) is not available, the dynamical renormalization group is able to resum the case $b_{k} \neq 0$.

\section{Case III: a logarithmically divergent spectral density at $\omega=\omega_{k}$.}

We now consider the case in which the spectral density is logarithmically divergent at $\omega_{k}$ but does not feature a normal threshold at this value of frequency, namely

$$
\rho_{k}^{(1)}(\omega) \stackrel{\omega \rightarrow \omega_{k}}{=} C_{k}\left[\log \left|\frac{\mu}{\omega-\omega_{k}}\right|+\mathcal{O}\left(\omega-\omega_{k}\right)\right] \quad ; \quad C_{k}>0 .
$$

In this case, using formulae (B.3)-B.4 from appendix B we find that the field expectation value takes the form of eq. (V.6) where the secular term $\mathcal{S}_{k}^{(1)}(t)$ is given by

$$
\lambda \mathcal{S}_{k}^{(1)}(t)=i\left[t \delta \omega_{k}-\frac{\lambda \pi C_{k}}{\omega_{k}^{2}} \ln \left(\mu t e^{\gamma-1}\right)\right]-\frac{\lambda \pi C_{k}}{2 \omega_{k}} t \ln \left[\mu t e^{\gamma-1}\right]
$$


Inserting eq. (V.26) in eq. (V.14 we find the DRG asymptotic long time behavior,

$$
\phi_{k}(t) \stackrel{t \rightarrow \infty}{=} \phi_{k}(0) \cos \left[\Omega_{k}(t)\right] e^{-\gamma_{k} t \ln [\bar{\mu} t]}+\text { c.c. },
$$

with

$$
\Omega_{k}(t)=\left(\omega_{k}+\delta \omega_{k}\right) t-\frac{\lambda \pi C_{k}}{\omega_{k}^{2}} \ln (\bar{\mu} t)
$$

and

$$
\gamma_{k}=\frac{\pi \lambda C_{k}}{2 \omega_{k}} \quad, \quad \bar{\mu}=\mu e^{\gamma-1}, \delta \omega_{k}=\frac{\lambda}{2 \omega_{k}} \operatorname{Re} \Sigma_{k}^{(1)}\left(\omega_{k}\right) .
$$

\section{E. Summary of dynamical renormalization group resummation to lowest order}

We are now in position to summarize the main results obtained to lowest order in the dynamical renormalization group resummation program.

The nature of the secular terms is completely determined by the behavior of the spectral density $\rho_{k}(\omega)$ near $\omega=\omega_{k}$. The different cases studied above to lowest order in the coupling $\lambda$ describe fairly general behavior of the spectral densities in quantum field theories at zero and non-zero temperature.

The important aspects of the results of this study can be highlighted as follows

- i): when the spectral density $\rho_{k}(\omega)$ vanishes at $\omega=\omega_{k}$ the particle is stable and the only effect of the interaction is to renormalize the mass and wave function. This corresponds to the spectral density of the particle featuring an isolated pole away from the branch cuts associated with the multiparticle production. The resummation implied by the dynamical renormalization group is equivalent to the Dyson resummation of the propagator that leads to the renormalized dispersion relation for the particle. When $\rho_{k}\left(\omega=\omega_{k}\right) \neq 0$ but $\omega_{k}>\omega_{t h}$ with $\omega_{t h}$ being the multiparticle thresholds, namely the beginning and end points of branch cuts in the spectral density of the particle, the particle is unstable and its propagator features a pole in an unphysical sheet in the complex $\omega$ plane, namely a resonance. In perturbation theory this resonance is very close to the real axis and the propagator is of the Breit-Wigner form. The dynamical renormalization group in this case provides a resummation which is equivalent to the Dyson series.

- ii): When the position of the multiparticle thresholds coincide with $\omega_{k}$, infrared divergences arise near the particle mass-shell. This corresponds to the emission and absorption of massless particles, a situation which is common in gauge theories and in theories with spontaneous symmetry breaking with Goldstone bosons. The secular terms that determine the relaxation of the mean field in perturbation theory depend on the behavior of the spectral density near thresholds. The dynamical renormalization group resummation in the case $\rho_{k}\left(\omega_{k} \pm 0\right)=0$ is similar to the Bloch-Nordsieck resummation of infrared divergences but directly in real time. No BlochNordsieck resummation is available for the case II, which we solve by DRG and where the spectral density is discontinuous $\left(\rho_{k}\left(\omega_{k}+0\right) \neq 0\right)$. Furthermore, the DRG provides a consistent resumation in the case III with a logarithmically divergent spectral density at $\omega=\omega_{k}$.

The secular terms associated with the different behaviors of the spectral density for these generic cases are summarized in the table below. 


\begin{tabular}{|c|c|c|c|c|}
\hline Case & $\rho_{k}(\omega) \stackrel{\omega \rightarrow \omega_{k}}{=}$ & Secular term $\mathcal{S}_{k}^{(1)}(t)$ & $\begin{array}{c}\text { DRG } \\
\text { resummed amplitude } \\
\delta(\vec{k}, t) \\
\end{array}$ & $\begin{array}{c}\begin{array}{c}\text { Resummation } \\
\text { done }\end{array} \\
\text { by the DRG }\end{array}$ \\
\hline I & $\begin{array}{c}\rho_{k}\left(\omega=\omega_{k}\right) \neq 0 ; \rho_{k}\left(\omega=\omega_{k} \pm 0\right) \neq 0 \\
\text { and continuous, } \omega_{k} \neq \omega_{t h}\end{array}$ & $\frac{t}{2 \omega_{k}}\left[i \operatorname{Re} \Sigma_{k}^{(1)}\left(\omega_{k}\right)+\operatorname{Im} \Sigma_{k}^{(1)}\left(\omega_{k}\right)\right]$ & $e^{i t\left(\omega_{k}+\delta \omega_{k}\right)-t \frac{\pi \rho_{k}\left(\omega_{k}\right)}{2 \omega_{k}}}$ & Dyson \\
\hline II & $\begin{array}{c}A_{k}\left[\omega-\omega_{k}+b_{k}\right] \Theta\left(\omega-\omega_{k}\right) \\
A_{k}, b_{k}>0\end{array}$ & $\begin{aligned} & \frac{i t}{2 \omega_{k}}\left[\operatorname{Re} \Sigma_{k}^{(1)}\left(\omega_{k}\right)-A_{k} b_{k} \ln \left(\omega_{k} t e^{\gamma-1}\right)\right] \\
- & \frac{A_{k}}{2 \omega_{k}}\left(1-\frac{2 b_{k}}{\omega_{k}}\right) \ln \left(\omega_{k} t e^{\gamma-1}\right)-t \frac{\pi \rho_{k}^{(1)}\left(\omega_{k}\right)}{4 \omega_{k}}\end{aligned}$ & $\begin{array}{c}e^{i t\left[\omega_{k}+\delta \omega_{k}-\lambda \frac{A_{k} b_{k}}{2 \omega_{k}} \ln \left(\omega_{k} t e^{\gamma-1}\right)\right.} \\
\times e^{-t \frac{\pi \rho_{k}\left(\omega_{k}\right)}{2 \omega_{k}}}\end{array}$ & \begin{tabular}{|c|} 
Dyson + \\
Bloch-Nordsieck \\
for the kernel \\
\end{tabular} \\
\hline III & $\begin{array}{c}C_{k} \ln \left|\frac{\mu}{\omega-\omega_{k}}\right| \\
C_{k}>0\end{array}$ & $\begin{array}{c}\frac{i}{2 \omega_{k}}\left[t \operatorname{Re} \Sigma_{k}\left(\omega_{k}\right)-\frac{2 \pi C_{k}}{\omega_{k}} \ln (\bar{\mu} t)\right] \\
-\frac{\pi C_{k}}{2 \omega_{k}} t \ln (\bar{\mu} t)\end{array}$ & $\begin{array}{c}e^{i t\left(\omega_{k}+\delta \omega_{k}\right)-\frac{\pi C_{k}}{2 \omega_{k}} t \log (\bar{\mu} t)} \\
\times(\bar{\mu} t)^{-\frac{i \pi C_{k}}{\omega_{k}^{2}}}\end{array}$ & \begin{tabular}{|c|} 
Dyson + \\
Bloch-Nordsieck \\
for the kernel
\end{tabular} \\
\hline
\end{tabular}




\section{SPECIFIC EXAMPLES IN A SCALAR THEORY:}

To highlight the main concepts in a simpler setting we study a scalar theory of three interacting fields $\Phi_{i} ; i=1,2,3$. The Lagrangian density is given by

$$
\mathcal{L}=\sum_{i=1}^{3}\left[\frac{1}{2}\left(\partial_{\mu} \Phi_{i}\right)^{2}-\frac{1}{2} m_{0, i}^{2} \Phi_{i}^{2}\right]-g_{o} \Phi_{1} \Phi_{2} \Phi_{3}+J_{o} \Phi_{1},
$$

where the source coupled to the field $\Phi_{1}$ has been introduced to provide an initial value problem for its expectation value as explained in section II, of course we could study simultaneously the initial value problem for the expectation values of all three fields, but our focus is to highlight the most important aspects in a simpler setting.

The renormalization is performed by introducing wave functions, mass and coupling renormalizations as follows

$$
\Phi_{i}=Z_{i}^{1 / 2} \Phi_{i, r} ; J_{r}=Z_{1}^{1 / 2} J_{o} \quad, \quad m_{0, i}^{2} Z_{i}=m_{r, i}^{2} Z_{i}+\delta m_{i}^{2} ; g_{o} \sqrt{Z_{1} Z_{2} Z_{3}}=g_{r} Z_{g} \quad, \quad i=1,2,3 .
$$

We now suppress the label $r$ with all quantities being renormalized, and write the Lagrangian density in terms of renormalized quantities and counterterms,

$$
\begin{aligned}
\mathcal{L} & =\sum_{i=1}^{3}\left[\frac{1}{2}\left(\partial_{\mu} \Phi_{i}\right)^{2}-\frac{1}{2} m_{i}^{2} \Phi_{i}^{2}\right]-g \Phi_{1} \Phi_{2} \Phi_{3}+J \Phi_{1}+\mathcal{L}_{c t} \\
\mathcal{L}_{c t} & =\sum_{i=1}^{3}\left[\frac{1}{2}\left(Z_{i}-1\right)\left(\partial_{\mu} \Phi_{i}\right)^{2}-m_{i}^{2} \Phi_{i}^{2}-\frac{1}{2} \delta m_{i}^{2} \Phi_{i}^{2}\right]-g\left(Z_{g}-1\right) \Phi_{1} \Phi_{2} \Phi_{3}
\end{aligned}
$$

The counterterms are adjusted in perturbation theory as usual.

Some cases which are relevant for the discussion that follows are:

- i: with $m_{1}>m_{2}+m_{3}$, in this case the decay and recombination processes $1 \leftrightarrow 2+3$ are allowed. This will lead to the presence of a resonance, namely the quasiparticle pole will be in the continuum resulting in a decay rate.

- ii: $m_{1}=m_{2} \neq 0 ; m_{3}=0$, this case will feature anomalous logarithmic relaxation as a consequence of the absorption and emission of soft massless quanta.

In what follows, we will treat both cases by setting $m_{3}=0$ and $m_{1}=m$ to present the main results in a simpler and more clear manner.

The calculation of the self-energy of particle 1 at finite temperature is best performed in the imaginary time formulation. The Matsubara Green's functions for the fields are written as spectral representations in the form

$$
G_{i}\left(k, \omega_{n}\right)=\frac{1}{\omega_{n}^{2}+\omega_{k, i}^{2}}=\int_{-\infty}^{+\infty} d p_{0} \frac{\rho_{i}\left(p_{0}, k\right)}{p_{0}-i \omega_{n}} ; \omega_{n}=2 \pi n T, \quad, \quad i=1,2,3 .
$$

with the spectral densities

$$
\rho_{i}\left(p_{0}, k\right)=\frac{1}{2 \omega_{k}}\left[\delta\left(p_{0}-\omega_{k, i}\right)-\delta\left(p_{0}+\omega_{k, i}\right)\right], \quad \omega_{k, i}^{2}=k^{2}+m_{i}^{2} \quad, \quad i=1,2,3 .
$$

The one-loop self-energy for the particle 1 is shown in figure 2 and is given by

$$
\Sigma_{1, k}\left(i \omega_{n}\right)=-g^{2} T \sum_{\omega_{m}} \int \frac{d^{3} p}{(2 \pi)^{3}} d p_{2} d p_{3} \frac{\rho_{2}\left(p_{2}, p\right)}{p_{2}-i \omega_{m}} \frac{\rho_{3}\left(p_{3},|\vec{p}+\vec{k}|\right)}{p_{3}-i \omega_{m}-i \omega_{n}}
$$

The sum over the Matsubara frequencies can be done using the methods described in [31, 32] leading to the following spectral representation for the self-energy

$$
\Sigma_{1, k}\left(i \omega_{n}\right)-\Sigma_{1, k}(0)=i \omega_{n} \int_{-\infty}^{+\infty} d \omega^{\prime} \frac{\rho_{k}\left(\omega^{\prime}\right)}{\omega^{\prime}\left(i \omega_{n}-\omega^{\prime}\right)},
$$




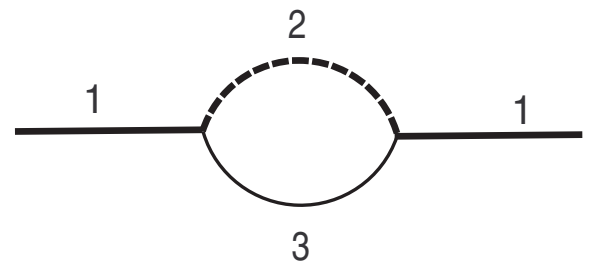

FIG. 2: One loop contribution to the self-energy of particle 1.

with the spectral density $\rho_{k}(\omega)$ given by

$\rho_{k}(\omega)=g^{2} \int \frac{d^{3} p}{(2 \pi)^{3}} d p_{2} d p_{3} \rho_{2}\left(p_{2}, p\right) \rho_{3}\left(p_{3},|\vec{p}+\vec{k}|\right)\left[n\left(p_{2}\right)-n\left(p_{3}\right)\right] \delta\left(p_{3}-p_{2}-\omega\right) ; n\left(p_{i}\right)=\frac{1}{e^{\frac{p_{i}}{T}}-1} \quad, \quad i=1,2,3$.

Comparing this dispersive representation for the self-energy with that of equation (III.7) we find that

$$
\Sigma_{k}(\omega)=\Sigma_{1, k}\left(i \omega_{n}=\omega+i 0\right)
$$

A straightforward but lengthy calculation with $m_{2}=M \neq 0 ; m_{3}=0$ leads to the following spectral density

$$
\begin{aligned}
\rho_{k}(\omega)= & \lambda m^{2}\left\{\left[\frac{\omega^{2}-W_{k}^{2}}{\omega^{2}-k^{2}}+\frac{T}{k} \log \left(\frac{1-e^{-u^{+}}}{1-e^{-u^{-}}} \frac{1-e^{-v^{+}}}{1-e^{-v^{-}}}\right)\right] \Theta\left(\omega^{2}-W_{k}^{2}\right)+\right. \\
& \left.+\frac{T}{k} \ln \left[\frac{1-e^{-u^{+}}}{1-e^{u^{-}}} \frac{1-e^{-v^{+}}}{1-e^{v^{-}}}\right] \Theta\left(k^{2}-\omega^{2}\right)\right\} \operatorname{sign}(\omega),
\end{aligned}
$$

with

$$
u^{ \pm}=\frac{\omega^{2}-W_{k}^{2}}{2 T(|\omega| \mp k)} \quad, \quad v^{ \pm}=\frac{|\omega|}{T}-u^{\mp} \quad, \quad W_{k}^{2}=k^{2}+M^{2}
$$

We identify the dimensionless coupling constant $\lambda \equiv \frac{g^{2}}{(2 \pi m)^{2}}$.

The contribution to the spectral density for $\omega^{2}>W_{k}^{2}$ corresponds to the two particle cut of one massive and one massless particle, and remains in the zero temperature limit. The contribution for $\omega^{2}<k^{2}$ corresponds to the Landau damping cut, only present at finite temperature and below the light cone.

This spectral density models all of the situations studied in the previous sections:

- Case I: $m \neq M$. In this case $\omega_{k}$ is away from the two particle threshold at $W_{k}$ and above the light cone, thus away from the Landau damping region. If $m<M$ the particle is stable and the self-energy correction amounts to a renormalization of the dispersion relation. If $m>M$ now $\omega_{k}$ lies in the two particle continuum, and the particle pole moves off the physical sheet, in this case the particle becomes a quasiparticle with a finite width $\propto \lambda$. In both cases $\rho_{k}\left(\omega=\omega_{k}\right) \neq 0$ and $\rho_{k}(\omega) \neq 0$ for $\omega \gtrless \omega_{k}$. This situation describes the case I analyzed in detail above and corresponds to the most common cases of stable particles or resonances.

- Case II: $m=M$. In this case the two particle threshold is precisely at $\omega_{k}$, but the two particle and Landau damping thresholds are well separated for any finite momentum $k$. The behavior of the spectral density near the threshold at $\omega_{k}$ is given by

$$
\rho_{k}(\omega) \stackrel{\omega \rightarrow \omega_{k}}{=} \lambda m^{2}\left[\frac{2 \omega_{k}}{m^{2}}\left(\omega-\omega_{k}\right)+\frac{T}{k} \ln \left|\frac{\omega_{k}+k}{\omega_{k}-k}\right|\right] \Theta\left(\omega-\omega_{k}\right) .
$$

This spectral density is of the same form as that of case II given by eq. $(\mathbb{V . 2 0})$. This case also applies to self energies of charged particles in gauge theories where the emission or absorption of massless gauge particles imply that $\omega_{k}$ is actually the beginning of a branch cut in the spectral representation of the charged particle propagator. While the (instantaneous) Coulomb interaction is screened at finite temperature by the polarization of the medium, transverse gluons (in perturbation theory) and photons are only dynamically screened by Landau 
damping 16, 17, 32]. Thus the self-energy of charged particles in general gauge theories features the threshold infrared divergences associated with case II and described by the threshold behavior given by eqs.(V.20) and (VI.12).

- Case III: $k>>m=M$, or $M=m=0$. In the case $M=m$ and for a hard particle $k>>m$ or alternatively for $M=m=0$, the behavior of the spectral density eq.(VI.10) above for $\omega_{k} \rightarrow k$ is seen to be of the form

$$
\rho_{k}(\omega) \stackrel{\omega \rightarrow k}{=} \lambda m^{2} \frac{T}{k} \ln \left|\frac{\mu(k)}{|\omega|-k}\right|
$$

where $\mu(k)=2 T\left(1-e^{-\frac{k}{T}}\right)$. In this case the Landau damping cut merges with the two particle cut since $\omega_{k}=k$ and the spectral density features a finite temperature logarithmic divergence at the position of the particle mass shell. This is the situation described by case III analyzed above and arises in the case of a hard charged particle, either fermionic or bosonic in high temperature gauge theories, where again the emission and absorption of transverse gauge fields, which are only dynamically screened introduces logarithmic divergences. In this case the notion of a damping rate which is associated with exponential relaxation is not the correct one to describe the relaxation of single quasiparticle excitations.

\section{TIME EVOLUTION IN QUANTUM MECHANICS}

While we have focused the discussion above on the evolution of the expectation value of quantum fields, the DRG approach also offers a systematic method to study the evolution of quantum states. In particular, as explained below in detail, the DRG offers an alternative to the adiabatic switching-on procedure to generate exact eigenstates of the interacting Hamiltonian from the eigenstates of the non-interacting system. Along the way this DRG formulation extracts the energy shifts, wave function renormalization constants and offers a clear description of Fermi's Golden rule.

Consider the time evolution of a quantum state in an interacting theory with Hamiltonian

$$
H=H_{0}+\lambda H_{I},
$$

namely

$$
|\psi(t)\rangle=e^{-i H\left(t-t_{0}\right)}\left|\psi\left(t_{0}\right)\right\rangle=e^{-i H_{0} t} U\left(t, t_{0}\right) e^{i H_{0} t_{0}}\left|\psi\left(t_{0}\right)\right\rangle .
$$

Where we have introduced the unitary time evolution operator in the interaction picture

$$
U\left(t, t_{0}\right)=1-i \lambda \int_{t_{0}}^{t} H_{I}\left(t^{\prime}\right) d t^{\prime}-\lambda^{2} \int_{t_{0}}^{t} \int_{t_{0}}^{t^{\prime}} H_{I}\left(t^{\prime}\right) H_{I}\left(t^{\prime \prime}\right) d t^{\prime} d t^{\prime \prime}+\mathcal{O}\left(\lambda^{3}\right) \quad, \quad U\left(t_{0}, t_{0}\right)=1,
$$

with the interaction Hamiltonian in the interaction picture

$$
H_{I}(t)=e^{i H_{0} t} H_{I} e^{-i H_{0} t} .
$$

It is convenient to pass to the interaction picture in which the states are given by

$$
|\psi(t)\rangle_{i}=e^{i H_{0} t}|\psi(t)\rangle
$$

and their time evolution is given by

$$
|\psi(t)\rangle_{i}=U\left(t, t_{0}\right)\left|\psi\left(t_{0}\right)\right\rangle_{i}
$$

Clearly, the time evolution of quantum states is an initial value problem.

The adiabatic, or Gell-Mann-Low theorem asserts that introducing the adiabatic time evolution operator $U_{\epsilon}\left(t, t_{0}\right)$ by replacing

$$
H_{I}(t) \rightarrow e^{-\epsilon|t|} H_{I}(t)
$$

in the time evolution operator $U\left(t, t_{0}\right)$ given by (VII.3), the states

$$
\left|\Psi_{n}\right\rangle=U_{\epsilon}(0,-\infty)|n\rangle,
$$


constructed out of the eigenstates $|n\rangle$ of the non-interacting Hamiltonian $H_{0}$ with energy $E_{n}$, are exact eigenstates of the total interacting Hamiltonian $H$, and the exact eigenvalues are then given by 33

$$
E_{n}+\Delta E_{n}=\frac{\left\langle\Psi_{n}|H| \Psi_{n}\right\rangle}{\left\langle\Psi_{n} \mid \Psi_{n}\right\rangle}
$$

We now show how the dynamical renormalization group provides an illuminating alternative to this procedure. To make this approach more clear and to establish contact with the familiar results, we now consider the case in which $\left|\psi\left(t_{0}\right)\right\rangle_{i}$ is an eigenstate of the non-interacting Hamiltonian $H_{0}$, namely

$$
\left|\psi\left(t_{0}\right)\right\rangle_{i}=|n\rangle ; H_{0}|n\rangle=E_{n}|n\rangle .
$$

To highlight the main ideas of the DRG, we will focus on the evolution of the (persistence) amplitude,

$$
C_{n}(t)=\langle n \mid \psi(t)\rangle_{i},
$$

however the time evolution of the off-diagonal overlaps $C_{m}(t)=\langle m \mid \psi(t)\rangle_{i} ; m \neq n$ can be studied along the same lines.

Inserting the identity $\sum_{m}|m\rangle\langle m|=1$ appropriately, we find for the amplitude $C_{n}(t)$ the following expression,

$$
\begin{aligned}
& C_{n}(t)=\left\langle n \mid U\left(t, t_{0}\right) \psi\left(t_{0}\right)\right\rangle_{i}=C_{n}\left(t_{0}\right)\left[1-i \lambda\left(t-t_{0}\right)\left\langle n\left|H_{I}\right| n\right\rangle-\frac{\lambda^{2}}{2}\left(t-t_{0}\right)^{2}\left\langle n\left|H_{I}\right| n\right\rangle^{2}\right. \\
& \left.-\lambda^{2} \int_{t_{0}}^{t} \int_{t_{0}}^{t^{\prime}} \sum_{m \neq n}\left|\left\langle n\left|H_{I}\right| m\right\rangle\right|^{2} e^{i\left(E_{n}-E_{m}\right)\left(t^{\prime}-t^{\prime \prime}\right)} d t^{\prime} d t^{\prime \prime}+\mathcal{O}\left(\lambda^{3}\right)\right],
\end{aligned}
$$

where we have written $C_{n}\left(t_{0}\right)=\left\langle n \mid \psi\left(t_{0}\right)\right\rangle_{i}$ despite our choice of initial state for which $C_{n}\left(t_{0}\right)=1$, to emphasize that the initial amplitude factors out.

It proves convenient to introduce the spectral density

$$
\rho_{n}(\omega)=\sum_{m \neq n}\left|\left\langle n\left|H_{I}\right| m\right\rangle\right|^{2} \delta\left(\omega-E_{m}\right),
$$

in terms of which and after the straightforward integration over the time variables $t^{\prime}, t^{\prime \prime}$, eq. (VII.12) becomes

$$
\begin{aligned}
& C_{n}(t)=C_{n}\left(t_{0}\right)\left\{1-i \lambda\left(t-t_{0}\right)\left\langle n\left|H_{I}\right| n\right\rangle-\frac{\lambda^{2}}{2}\left(t-t_{0}\right)^{2}\left\langle n\left|H_{I}\right| n\right\rangle^{2}\right. \\
& \left.-\lambda^{2} \int d \omega \rho_{n}(\omega)\left[\frac{i}{E_{n}-\omega}\left(t-t_{0}-\frac{\sin \left[\left(E_{n}-\omega\right)\left(t-t_{0}\right)\right]}{E_{n}-\omega}\right)+\frac{1-\cos \left[\left(E_{n}-\omega\right)\left(t-t_{0}\right)\right]}{\left(E_{n}-\omega\right)^{2}}\right]+\mathcal{O}\left(\lambda^{3}\right)\right\} .
\end{aligned}
$$

Using formulae (B.1) and (B.5) of appendix B we find the asymptotic long time limit to be given by,

$$
\begin{aligned}
C_{n}(t)= & C_{n}\left(t_{0}\right)\left\{1-i \lambda\left(t-t_{0}\right)\left\langle n\left|H_{I}\right| n\right\rangle-\frac{\lambda^{2}}{2}\left(t-t_{0}\right)^{2}\left\langle n\left|H_{I}\right| n\right\rangle^{2}-i\left(t-t_{0}\right) \lambda^{2} \sum_{m}^{\prime} \frac{\left|\left\langle n\left|H_{I}\right| m\right\rangle\right|^{2}}{E_{n}-E_{m}}\right. \\
& \left.-\lambda^{2} \pi\left(t-t_{0}\right) \rho_{n}\left(E_{n}\right)-\lambda^{2} \sum_{m}^{\prime} \frac{\left|\left\langle n\left|H_{I}\right| m\right\rangle\right|^{2}}{\left(E_{n}-E_{m}\right)^{2}}+\mathcal{O}\left(\frac{1}{t-t_{0}}, \lambda^{3}\right)\right\} .
\end{aligned}
$$

where $\sum_{m}{ }^{\prime}$ refers to the sum over all the states with $E_{m} \neq E_{n}$. We write the expression above as,

$$
C_{n}(t)=C_{n}\left(t_{0}\right)\left[1+\lambda \mathcal{S}_{n}^{(1)}(t)+\lambda^{2} \mathcal{S}_{n}^{(2)}(t)-\lambda^{2} \mathcal{Z}_{n}^{(2)}+\mathcal{O}\left(\frac{1}{t-t_{0}}, \lambda^{3}\right)\right]
$$

where $\lambda^{(i)} \mathcal{S}_{i}^{(i)}(t)$ are secular terms (linear and quadratic in time respectively), the term $-\lambda^{2} \mathcal{Z}_{n}^{(2)}$ in eq. (VII.16) is the time independent term in eq. (VII.15).

Applying here the dynamical renormalization group, we introduce the amplitude renormalization in the form

$$
C_{n}\left(t_{0}\right)=C_{n}(\tau) R_{n}(\tau) \quad ; \quad R_{n}(\tau)=1+\lambda r_{n}^{(1)}(\tau)+\lambda^{2} r_{n}^{(2)}(\tau)+\mathcal{O}\left(\lambda^{3}\right)
$$


Hence,

$$
C_{n}(t)=C_{n}(\tau)\left\{1+\lambda\left[r_{n}^{(1)}(\tau)+\mathcal{S}_{n}^{(1)}(t)\right]+\lambda^{2}\left[r_{n}^{(2)}(\tau)+\mathcal{S}_{n}^{(2)}(t)+r_{n}^{(1)}(\tau) \mathcal{S}_{n}^{(1)}(t)\right]-\lambda^{2} \mathcal{Z}_{n}^{(2)}+\mathcal{O}\left(\lambda^{3}\right)\right\}
$$

The counterterms $r_{n}^{(i)}(\tau)$ are required to cancel the secular terms at a time scale $t=\tau$, leading to

$$
r_{n}^{(1)}(\tau)=-\mathcal{S}_{n}^{(1)}(\tau) \quad, \quad r_{n}^{(2)}(\tau)=-\mathcal{S}_{n}^{(2)}(\tau)+\left[r_{n}^{(1)}(\tau)\right]^{2} .
$$

The independence of the amplitude $C_{n}(t)$ on the arbitrary time scale $\tau$, namely $d C_{n}(t) / d \tau=0$ leads to the dynamical renormalization group equation

$$
\dot{C}_{n}(\tau)\left\{1+\lambda\left[r_{n}^{(1)}(\tau)+\mathcal{S}_{n}^{(1)}(t)\right]+\mathcal{O}\left(\lambda^{3}\right)\right\}+C_{n}(\tau)\left\{\lambda \dot{r}_{n}^{(1)}(\tau)+\lambda^{2}\left[\dot{r}_{n}^{(2)}(\tau)+\dot{r}_{n}^{(1)}(\tau) \mathcal{S}_{n}^{(1)}(t)\right]+\mathcal{O}\left(\lambda^{3}\right)\right\}=0
$$

where the dot stands for derivative with respect to $\tau$. Keeping terms up to second order in $\lambda$ yields,

$$
\frac{\dot{C}_{n}(\tau)}{C_{n}(\tau)}=-\frac{d}{d \tau}\left\{\lambda r_{n}^{(1)}(\tau)+\lambda^{2}\left[r_{n}^{(2)}(\tau)-\frac{1}{2}\left(r_{n}^{(1)}(\tau)\right)^{2}\right]\right\}+\mathcal{O}\left(\lambda^{3}\right)
$$

Notice that the $t$-dependent pieces cancelled out identically. Such cancellation is necessary for the consistency of the DRG to second order in $\lambda$. We find using the counterterms given by eq.(VII.19),

$$
C_{n}(\tau)=C_{n}\left(t_{0}\right) e^{\lambda S_{n}^{(1)}(\tau)+\lambda^{2}\left\{\mathcal{S}_{n}^{(2)}(\tau)-\frac{1}{2}\left[S_{n}^{(1)}(\tau)\right]\right\}} .
$$

Using now the explicit form of the secular terms which are read off from eq.(VII.15), we finally find the DRG solution to second order to be given by

$$
C_{n}(\tau)=C_{n}(0) e^{-i \Delta E_{n} \tau} e^{-\frac{\Gamma n \tau}{2}},
$$

with the energy shift and width up to $\mathcal{O}\left(\lambda^{2}\right)$ given by

$$
\Delta E_{n}=\lambda\left\langle n\left|H_{I}\right| n\right\rangle+\lambda^{2} \sum_{m}^{\prime} \frac{\left|\left\langle n\left|H_{I}\right| m\right\rangle\right|^{2}}{E_{n}-E_{m}} \quad, \quad \Gamma_{n}=2 \pi \rho_{n}\left(E_{n}\right) .
$$

Notice that the terms in $\tau^{2}$ cancel identically in the exponent of eq.(VII.21) and (VII.22). Again, such cancellation is necessary for the consistency of the DRG.

Finally, inserting eq.(VII.22) into eq. (VII.18) and choosing the arbitrary renormalization scale $\tau$ to coincide with the time $t$, we find the asymptotic long time behavior of the dynamical renormalization group resummed amplitude as

$$
C_{n}(t) \stackrel{t \rightarrow \infty}{=} \mathcal{Z}_{n} e^{-i \Delta E_{n} t} e^{-\frac{\Gamma n t}{2}} .
$$

The energy shift $\Delta E_{n}$ is clearly the same obtained in familiar perturbation theory, the decay rate $\Gamma_{n}$ coincides with the transition probability per unit time obtained from Fermi's Golden rule, and the wave function renormalization

$$
\mathcal{Z}_{n}=1-\lambda^{2} \sum_{m}^{\prime} \frac{\left|\left\langle n\left|H_{I}\right| m\right\rangle\right|^{2}}{\left(E_{n}-E_{m}\right)^{2}}=\frac{\partial}{\partial E_{n}}\left(E_{n}+\Delta E_{n}\right)
$$

is the same as obtained in usual perturbation theory and determines the overlap between the unperturbed state and the exact eigenstate of the Hamiltonian [33]. Thus, we see that the dynamical renormalization group leads to an alternative formulation of the construction of the exact eigenstates which allows to extract the energy shifts, widths and weights and that coincides with the familiar setting of quantum mechanics.

There is an important byproduct of this exercise: by considering the perturbative expansion up to second order in the interaction Hamiltonian, we found secular terms that contain the square of the first order secular terms. These must cancel consistently and systematically in the final DRG equation since they are accounted for in the resummation furnished by the DRG. Indeed we found such cancellation in eqs.(VII.21) and (VII.22). This is the equivalent of the renormalizability of the perturbative expansion. Thus, this example not only provides a pedagogical framework to explore and confirm the dynamical renormalization group, but also manifestly shows the renormalizability in the sense that higher order terms that appear in the perturbative expansion, which are associated with the expansion of first order terms in the solution, cancel systematically in the dynamical renormalization group equation. 


\section{DYNAMICAL RENORMALIZATION GROUP: RESUMMATIONS}

The examples studied in detail above clearly indicate that the dynamical renormalization group provides a resummation of the perturbative series. Thus the following question arises : which type of resummation is implemented by the DRG?. The answer to this important question can be gleaned from the examples corresponding to the different cases discussed in the previous sections.

- Case I: in the case in which the position of the particle mass shell near $\omega_{k}$ is far away from the multiparticle thresholds in the perturbative expansion, then the DRG provides a resummation which is akin to the geometric Dyson sum of the propagator with the perturbative self-energy but keeping only the secular terms at each order. The DRG yields the asymptotic long time behavior of the expectation value of the field in the case of stable particles or unstable quasiparticles with a lifetime (width or decay rate) determined by the spectral density at the position of the mass shell of the particle.

- Cases II and III: In the case in which there are threshold divergences in the propagator corresponding to the case when the position of the particle mass shell at $\omega=\omega_{k}$ coincides with the beginning (or end point) of a branch cut, the DRG provides a resummation of the self-energy kernel along with a resummation of the perturbative series for the propagator à la Dyson. That this is indeed the case can be understood from the study in section IVC where Bloch-Nordsieck resummation of the propagator exponentiates the logarithmic threshold divergences. This resummation of the propagator is akin to that via the usual Euclidean renormalization group and leads to a power law relaxation, just as the scaling behavior of renormalization group improved correlation functions. The case of threshold divergences at finite temperature is much more severe by the enhancement of the infrared behavior by the Bose-Einstein occupation factors. In this case there is no readily available Bloch-Nordsieck or renormalization group resummation for the Euclidean propagator, but the DRG furnishes the resummation directly in real time. Thus, in conclusion, in all of these cases in which there are infrared divergences, typically arising from the emission and absorption of massless quanta, the DRG provides a resummation of the self-energy kernel along with the Dyson type resummation of the perturbative series.

It is important to highlight that the DRG does all of these resummations automatically. Whereas in the usual approach, there is first the resummation of the Dyson series of the propagator, and in the case of threshold divergences the self-energy has to be further resummed independently, either by the Bloch-Nordsieck method or alternatively by the Euclidean renormalization group. Thus, insofar as extracting the asymptotic long time behavior of the expectation values, the DRG provides a powerful, systematic method with a straightforward implementation which furnishes several stages of resummation within a single approach.

Secular terms and separation of time scales: For any finite time the perturbative expansion is well defined and is free of any divergences (ultraviolet divergences had been properly renormalized). It is for long times that secular terms that grow in time invalidate the perturbative expansion. Consider the example of case I where the solution to first order in the coupling is given by eq.(V.16). It is clear from this expression that the perturbation expansion breaks down at a time scale $t_{b}$ when

$$
\lambda t_{b}\left|\frac{i \operatorname{Re} \Sigma_{k}^{(1)}\left(\omega_{k}\right)}{2 \omega_{k}}-\frac{\pi \rho_{k}^{(1)}\left(\omega_{k}\right)}{2 \omega_{k}}\right| \sim 1 .
$$

This time scale is obviously of the same order as the relaxation time $t_{b} \simeq t_{r e l}=\Gamma_{k}^{-1}$ where $\Gamma_{k}$ is the width given by eq.(V.18). Secular terms dominate the integral in eq. (V.5 for sufficiently late times. Let $t_{\rho}$ be such time scale, namely, for $t \geq t_{\rho}$ we can approximate the integral in eq. the explicit examples discussed above, the nature of the secular terms as well as the scale $t_{\rho}$ depend on the behavior of the spectral density near $\omega=\omega_{k}$. The detailed behavior of the spectral density depends both on the field theory studied as well as the properties of the medium or the initial state chosen.

Thus, the perturbative expansion features secular terms but it is still reliable in a time interval such that

$$
t_{\rho} \ll t \ll t_{\text {rel }} .
$$

Therefore, if there is a wide separation of time scales such that $t_{r e l} \gg t_{\rho}$, then, there is a wide region in time during which secular terms can be clearly identified and yet the perturbative expansion is valid. The secular terms can be extracted and renormalized during this large interval and the DRG resummation extends the result uniformly for $t \gg t_{r e l}$, namely beyond the validity of the original perturbative expansion.

Since the spectral densities do not depend on the coupling (to lowest order) but the relaxation time scale is such that $t_{r e l} \sim 1 /[m \lambda]$ a wide separation of time scales is justified in the weak coupling limit. In higher orders the width 
is always at least one more power of the coupling as compared to the spectral density. Hence, if there is a separation of scales in lowest order, such separation will remain in higher orders.

Thus, the conclusion of this discussion is that the DRG allows a systematic improvement of the perturbative series, leading to a uniform expansion, in a weakly coupled theory in which a wide separation between the typical time scale beyond which there emerge secular terms and the relaxation time scale is justified.

\section{DRG and Euclidean RG:}

The connection with the more familiar Euclidean renormalization group can be established with the following

example. Consider a theory of a single scalar field $\phi$ with a quartic self-interaction $\mathcal{L}_{\text {int }}=-\frac{\lambda_{0}}{4 !} \phi^{4}$ in four space-time dimensions defined with an upper momentum cutoff $\Lambda$. The $2 \rightarrow 2$ particle scattering amplitude with all equal external momentum is given by

$$
\Gamma^{(4)}(p, p, p, p)=\lambda_{0}-\frac{3}{2} \lambda_{0}^{2} t \quad, \quad t=\ln \left(\frac{\Lambda}{p}\right) .
$$

Thus we see that the usual logarithmic divergences in the scattering amplitude can be interpreted as secular terms in the perturbative expansion.

Introducing $\lambda_{R}[\kappa]$, the coupling renormalized at a scale $\kappa$ as

$$
\lambda_{0}=\lambda_{R}[\kappa] Z_{\lambda}[\kappa] ; Z_{\lambda}[\kappa]=1+\lambda_{R} z_{1}[\kappa]+\mathcal{O}\left(\lambda^{2}\right)
$$

and choosing $z_{1}[\kappa]=\ln \left(\frac{\Lambda}{\kappa}\right)=\tau$ to cancel the secular term in the variable $t$ in the scattering amplitude at the scale $p=\kappa$, we find to second order in the renormalized coupling

$$
\Gamma^{(4)}(p, p, p, p)=\lambda_{R}[\kappa]+\frac{3}{2} \lambda_{R}^{2}[\kappa](\tau-t) \quad, \quad t=\ln \left(\frac{\Lambda}{p}\right) \quad, \quad \tau=\ln \left(\frac{\Lambda}{\kappa}\right) .
$$

Since the scattering amplitude cannot depend on the arbitrary renormalization scale $\kappa$, namely

$$
\frac{d}{d \tau} \Gamma^{(4)}(p, p, p, p)=0
$$

the renormalization group equation for the coupling follows, namely

$$
\dot{\lambda}_{R}+\frac{3}{2} \lambda_{R}^{2}=0 \Rightarrow \beta_{\lambda}=-\frac{3}{2} \lambda_{R}^{2} ; \beta_{\lambda}=\kappa \frac{\partial}{\partial \kappa} \lambda_{R}[\kappa]
$$

Solving this renormalization group equation, where $\beta_{\lambda}$ is recognized as the usual renormalization group beta function, and choosing the scale $\tau$ to coincide with $t$, we obtain the renormalization group improved scattering amplitude

$$
\Gamma^{(4)}(p, p, p, p)=\frac{\lambda_{R}[\kappa]}{1+\frac{3}{2} \lambda_{R}[\kappa] \ln \left(\frac{\kappa}{p}\right)} .
$$

This expression reveals that the renormalization group is resumming the geometric series of one loop bubbles but only keeping the secular terms in the loops, namely the logarithmic terms. This resummation is tantamount to summing the leading logarithms.

\section{SUMMARY AND CONCLUSIONS}

In this article we studied the real time evolution and relaxation of expectation values of quantum fields as well as the evolution of the states in quantum mechanics by implementing the dynamical renormalization group.

The time evolution of expectation values of a bosonic quantum field is studied as an initial value problem. The fully renormalized equation of motion for the expectation value is obtained in linear response to an external current that is adiabatically switched on. We first studied the time evolution in terms of the Laplace transform of the equation of motion with a one-loop retarded self-energy, which is tantamount to a Dyson resummation (geometric series) of the self-energy. The real time evolution was obtained in several cases of interest: stable particles and quasiparticles (resonances) and in the case in which the propagator features threshold infrared divergences, which are a consequence of absorption and emission of massless quanta at zero or non-zero temperature. These threshold divergences invalidate the perturbative expansion and are ubiquitous in gauge theories. A Bloch-Nordsieck resummation leads to anomalous 
relaxation in the case of logarithmic infrared singularities but is not readily available in the case of more severe infrared singularities.

A strict perturbative expansion of the solution of the equations of motion (III.5 features secular terms, namely terms that grow in time and invalidate the perturbative expansion. We straightforwardly obtain the perturbative solution of the equations of motion by expanding the Laplace transform solution eqs. (III.18)-(III.19) in powers of $\lambda$. One can alternatively solve by iteration the evolution equations (III.5) 18].

We then implemented the dynamical renormalization group (DRG) to resum these secular terms in all cases. The DRG effectively resums the self-energy kernel of the evolution equations (III.5) while the Laplace transform eqs. (III.18)-(III.19) provides the exact solution with the input kernel (which is to first order in $\lambda$ here.) The DRG method provides a consistent and systematic approach that at once resums the secular terms. The DRG solution eq. (V.14 is a uniform asymptotic expansion for late times.

The secular terms are linear in time in the case of stable particles or quasiparticles and logarithmic in time or linear times logarithmic in the case of threshold infrared divergences. In the case of stable particles and quasiparticles (resonances) the resummation implied by the DRG is the same as that of the Dyson series or Laplace transform. However, in the case of threshold infrared divergences the DRG provides at once a Bloch-Nordsieck type of resummation of the self-energy kernel and a Dyson series resummation of the solution.

We then studied the time evolution in quantum mechanics as an initial value problem for the states by implementing the dynamical renormalization group. Again, the perturbative expansion features secular terms, which upon resummation via the DRG allow to extract the energy shift and width of quantum levels as well as the wave function renormalizations. Thus, the DRG provides a framework to systematically obtain energy shifts and width of quantum states. The consistency of the DRG to the second order of perturbations is proved here. Such consistency is analogous to the renormalizability of quantum field theory for the renormalization group.

While we discussed primarily the case of bosonic theories, the method can be simply generalized to fermionic and gauge theories $[18,19]$.

There are many distinct advantages to this method that make it a very powerful way to study relaxation directly in real time. While the most often used description of relaxation is based on the concept of quasiparticle widths and is associated with exponential relaxation, the DRG does not bias the description: if the secular terms are linear in time, indeed the concept of a quasiparticle width is the relevant one. On the other hand if the secular terms are more complicated, for example logarithmic or linear in time times logarithmic, the concept of a quasiparticle width and exponential relaxation is not the proper one. The dynamical renormalization group leads to the correct relaxational dynamics either power laws in the case of logarithmic secular terms or more complicated exponentials in the case of linear times logarithmic. These relaxational dynamics simply cannot be extracted from the usual concept of quasiparticle widths which are infrared divergent 16, 17].

Thus, one of the main conclusions of this article is that the dynamical renormalization group provides a powerful, systematic and easy to implement framework to study the relaxational dynamics of expectation values and the time evolution of quantum states directly in real time. This method implements a resummation of the perturbative expansion which yields a uniform asymptotic long time behavior and reveals the correct relaxational dynamics, all within one simple method.

While we have illustrated the main aspects of the DRG to lowest order in the perturbative expansion, the next step is to extend the DRG calculations to higher loops in field theory.

\section{ACKNOWLEDGMENTS}

We thank Emil Mottola for probing questions and useful discussions. The work of D.B. was supported in part by the US National Science Foundation under grants PHY-9988720 and NSF-INT-9905954.

\section{APPENDIX A: RENORMALIZED EQUATION OF MOTION AND ITS LAPLACE TRANSFORM}

The wave function and mass renormalization [eq.(III.3)] are defined by the counterterms $\delta m^{2}$ and $Z$ which are consistently computed in a perturbative expansion in terms of the coupling constant. Hence, the renormalized retarded self-energy is related to the bare one by

$$
\widetilde{\Sigma}_{k}(s) \equiv(Z-1)\left(s^{2}+\omega_{k}^{2}\right)+\widetilde{\Sigma}_{k, \text { bare }}(s)+\delta m^{2}
$$

Using this renormalized self-energy the tadpole condition eq.(III.4) leads to the equation of motion (III.5). 
We perform now the Laplace transform of eq.(III.5). We find,

$$
\int_{0}^{\infty} d t e^{-s t} \ddot{\phi}_{k}(t)=s^{2} \varphi_{k}(s)-\dot{\phi}_{k}(0)-s \phi_{k}(0) .
$$

In the Laplace transform of the kernel term we change the integration variable $t^{\prime}$ by $x \equiv t-t^{\prime}>0$ and then the integration variable $t$ by $y \equiv t-x$. We obtain,

$$
\begin{aligned}
& \int_{0}^{\infty} d t e^{-s t} \int_{-\infty}^{t} d t^{\prime} \Sigma_{k}\left(t-t^{\prime}\right) \phi_{k}\left(t^{\prime}\right)=\int_{0}^{\infty} d t e^{-s t} \int_{0}^{\infty} d x \Sigma_{k}(x) \phi_{k}(t-x)= \\
& =\int_{0}^{\infty} d x e^{-s x} \Sigma_{k}(x) \int_{-x}^{\infty} d y e^{-s y} \phi_{k}(y)=\int_{0}^{\infty} d x e^{-s x} \Sigma_{k}(x)\left[\frac{e^{s x}-1}{s} \phi_{k}(0)+\int_{0}^{\infty} d y e^{-s y} \phi_{k}(y)\right]= \\
& =\frac{\phi_{k}(0)}{s}\left[\tilde{\Sigma}_{k}(0)-\tilde{\Sigma}_{k}(s)\right]+\tilde{\Sigma}_{k}(s) \varphi_{k}(s)
\end{aligned}
$$

where we used that $\phi_{k}(t)=\phi_{k}(0)$ for $t<0$.

\section{APPENDIX B: ASYMPTOTIC BEHAVIOR OF SPECTRAL INTEGRALS}

We summarize in this appendix the late time behavior of integrals over the density of states used in Sections III.

In the formulas below $p(y)$ stands for a smooth function for $0 \leq y \leq \infty . p(0)$ and $p^{\prime}(0)$ are finite. For large $y, p(y)$ are assumed to fall so that the integrals over $y$ converge at infinity.

$$
\begin{gathered}
\int_{-A}^{\infty} \frac{d y}{y^{2}}(1-\cos y t) p(y) \stackrel{t \rightarrow \infty}{=} \pi t p(0)+\mathcal{P} \int_{-A}^{\infty} \frac{d y}{y^{2}} p(y)+\mathcal{O}\left(\frac{1}{t}\right) \\
\int_{0}^{\infty} \frac{d y}{y}(1-\cos y t) p(y) \stackrel{t \rightarrow \infty}{=} p(0)[\ln (\mu t)+\gamma]+\int_{0}^{\infty} \frac{d y}{y}[p(y)-p(0) \theta(\mu-y)]+\mathcal{O}\left(\frac{1}{t}\right) \\
\int_{-A}^{\infty} \frac{d y}{y^{2}}(1-\cos y t) p(y) \ln \frac{|y|}{\mu} \stackrel{t \rightarrow \infty}{=} \pi t p(0)[1-\gamma-\ln (\mu t)]+\int_{-A}^{\infty} \frac{d y}{y^{2}} p(y) \ln \frac{|y|}{\mu}+\mathcal{O}\left(\frac{1}{t}\right) \\
\int_{-A}^{\infty} \frac{d y}{y}\left(t-\frac{\sin y t}{y}\right) p(y) \ln \frac{|y|}{\mu} \stackrel{t \rightarrow \infty}{=} t \mathcal{P} \int_{-A}^{\infty} \frac{d y}{y} p(y) \ln \frac{|y|}{\mu}-\pi p^{\prime}(0)[\ln (\mu t)+\gamma]+\mathcal{O}\left(\frac{1}{t}\right) \\
\int_{-A}^{\infty} \frac{d y}{y}\left(t-\frac{\sin y t}{y}\right) p(y) \stackrel{t \rightarrow \infty}{=} t \mathcal{P} \int_{-A}^{\infty} \frac{d y}{y} p(y)-\pi p^{\prime}(0)+\mathcal{O}\left(\frac{1}{t}\right) \\
\int_{0}^{\infty} \frac{d y}{y^{2}}(1-\cos y t) p(y) \stackrel{t \rightarrow \infty}{=} \frac{\pi}{2} t p(0)+p^{\prime}(0)[\ln (\mu t)+\gamma] \\
\int_{0}^{\infty} \frac{d y}{y}\left(t-\frac{\sin y t}{y}\right) p(y) \stackrel{t \rightarrow \infty}{=} t p(0)[\ln (\mu t)+\gamma-1]+t \int_{0}^{\infty} \frac{d y}{y}[p(y)-p(0) \theta(\mu-y)]+\mathcal{O}\left(\frac{1}{t}\right)
\end{gathered}
$$

where $A$ is a fixed positive number and $\gamma=0.5772157 \ldots$ is Euler's constant and $\mathcal{P}$ stands for the principal part.

Notice that the formulas are independent of the scale $\mu$ as one can easily see since the derivative with respect to $\mu$ of the r. h. s. identically vanishes. The scale $\mu$ has been introduced just to have a dimensionless argument in the $\operatorname{logs}$.

\section{APPENDIX C: THE DYNAMICAL RENORMALIZATION GROUP IN A SOLVABLE EXAMPLE}

In this appendix we present the dynamical renormalization group through a simple example chosen for pedagogical reasons which allows to illustrate the fundamental features within a simple setting.

We consider a damped harmonic oscillator:

$$
\ddot{y}+y=-\epsilon \dot{y}, \epsilon \ll 1 \text {. }
$$


This equation admits as exact solution,

$$
y(t)=a e^{-\frac{\epsilon}{2} t} \cos \left(t \sqrt{1-\frac{\epsilon^{2}}{4}}+b\right)
$$

where the constants $a$ and $b$ are determined by the initial conditions.

We seek a solution as a perturbative expansion in $\epsilon$, of the form

$$
y(t)=y_{0}(t)+\epsilon y_{1}(t)+\epsilon^{2} y_{2}(t)+\mathcal{O}\left(\epsilon^{3}\right)
$$

where the $y_{i}(t)$ are solutions to the following hierarchy of equations:

$$
\ddot{y}_{0}(t)+y_{0}(t)=0 \quad, \quad \ddot{y}_{1}(t)+y_{1}(t)=-\dot{y}_{0}(t) \quad, \quad \ddot{y}_{2}(t)+y_{2}(t)=-\dot{y}_{1}(t) \quad, \quad \cdots
$$

These equations can be solved iteratively by starting from the zero order solution

$$
y_{0}(t)=A e^{i t}+\text { c.c }
$$

using the retarded Green's function

$$
G_{\text {ret }}(t)=\theta(t) \sin t
$$

which is the solution of differential equation

$$
\left[\frac{d^{2}}{d t^{2}}+1\right] G_{\mathrm{ret}}(t)=\delta(t)
$$

Up to second order in $\epsilon$, the perturbative expansion of the solution is given by

$$
y(t)=A e^{i t}\left[1-\frac{\epsilon}{2} t+\frac{\epsilon^{2}}{8} t^{2}+i \frac{\epsilon^{2}}{8} t\right]+\text { c.c. }+ \text { non-secular }
$$

Note that this solution contains secular terms that grow in $t$, the terms denoted by non-secular remain finite at all times. We see that this asymptotic expansion is only valid for $\epsilon t \ll 1$.

The dynamical renormalization is achieved by introducing the renormalization constant $Z(\tau)$ in the form

$$
A=A(\tau) Z(\tau)
$$

with the expansion

$$
Z(\tau)=1+\epsilon z_{1}(\tau)+\epsilon^{2} z_{2}(\tau)+\mathcal{O}\left(\epsilon^{3}\right)
$$

The coefficients $z_{i}(\tau)$ are chosen to cancel the secular terms at a the time scale $t=\tau$. This is similar to choosing the wave function renormalization in field theory to absorb the UV (or IR) divergences at a given momentum scale. Up to $\mathcal{O}\left(\epsilon^{2}\right)$ we find

$$
z_{1}(\tau)=\frac{\tau}{2} \quad ; \quad z_{2}(\tau)=\frac{\tau^{2}}{8}-i \frac{\tau}{8}
$$

After this renormalization the solution (C.4) takes the form,

$$
y(t, \tau)=A(\tau) e^{i t}\left[1-\frac{\epsilon}{2}(t-\tau)+\frac{\epsilon^{2}}{8}(t-\tau)^{2}+i \frac{\epsilon^{2}}{8}(t-\tau)\right]+\text { c.c. }+ \text { nonsecular } .
$$

We have now an expansion in powers of $\epsilon|t-\tau|$. This asymptotic expansion is expected to be valid for $\epsilon|t-\tau| \ll 1$. That is, renormalization has improved the validity region of the solution from the neighborhood of $t=0$ [see eq. (C.4)] to the neighborhood of $t=\tau$ which is an arbitrary point.

Since $\tau$ is an arbitrary scale, the solution must not depend on it. We therefore request

$$
\frac{d y(t, \tau)}{d \tau}=0 .
$$


This condition leads to the following equation to this order

$$
\frac{\partial A(\tau)}{\partial \tau}+\left(\frac{\epsilon}{2}-i \frac{\epsilon^{2}}{8}\right) A(\tau)=0 .
$$

where we have neglected terms of order higher than $\epsilon^{2}$. Eq. (C.6. is the dynamical renormalization group equation to this order. Eq. Can be easily solved with the solution

$$
A(\tau)=A(0) e^{-\frac{\epsilon}{2} \tau+i \frac{\epsilon^{2}}{8} \tau} .
$$

Since the solution $y(t, \tau)$ is independent of $\tau$ thanks to eq. (C.5. we finally set $t=\tau$ which yields,

$$
y(t)=A(0) e^{-\frac{\epsilon}{2} t+i\left(1-\frac{\epsilon^{2}}{8}\right) t}+\text { c.c. }
$$

Comparing eq.(C.7) with eq.(C.2) shows that eq.(C.7) provides an uniform approximation to the exact solution (C.2) up to $\mathcal{O}\left(\epsilon^{3}\right)$. That is, the dynamical renormalization group solution (C.7) differs from the exact solution (C.2) by $\mathcal{O}\left(\epsilon^{3}\right)$ whatever is $t$.

The solution (C.7) is nothing but an envelope of the family of solutions (C.4) parametrized by $\tau$ [21]. In other words, the $\tau$-family (C.4) provides a solution for $t$ near $\tau$ while eq.(C.7) is a good approximation valid for all $t$.

Further examples can be found in refs. 20, 21, 22].

[1] J. W. Harris and B. Muller, Annu. Rev. Nucl. Part. Sci. 46, 71 (1996). B. Muller in Particle Production in Highly Excited Matter, Eds. H.H. Gutbrod and J. Rafelski, NATO ASI series B, vol. 303 (1993). B. Muller, The Physics of the Quark Gluon Plasma Lecture Notes in Physics, Vol. 225, Springer-Verlag, Berlin, Heidelberg, 1985; B. Muller, The Physics of the Quark Gluon Plasma Lecture Notes in Physics, Vol. 225, Springer-Verlag, Berlin, Heidelberg, 1985.

[2] H. Meyer-Ortmanns, Rev. of Mod. Phys. 68, 473 (1996).

[3] H. Satz, in Proceedings of the Large Hadron Collider Workshop ed. G. Jarlskog and D. Rein (CERN, Geneva), Vol. 1. page 188; and in Particle Production in Highly Excited Matter, Eds. H.H. Gutbrod and J. Rafelski, NATO ASI series B, vol. 303 (1993).

[4] K. Geiger, Phys. Rep. 258, 237 (1995); Phys. Rev. D46, 4965 (1992); Phys. Rev. D47, 133 (1993); Quark Gluon Plasma 2, Ed. by R. C. Hwa, World Scientific, Singapore, 1995.

[5] X. N. Wang, Phys. Rep. 280, 287 (1997).

[6] M. H. Thoma, in Quark Gluon Plasma 2, ed. by R. C. Hwa, World Scientific, Singapore, 1995.

[7] J.-P Blaizot, J. -Y. Ollitrault and E. Iancu, in Quark Gluon Plasma 2, Ed. by R. C. Hwa, World Scientific, Singapore, 1995.

[8] H. Elze and U. Heinz, Phys. Rep. 183, 81 (1989).

[9] E. W. Kolb and M. S. Turner, The Early Universe, Addison-Wesley Pub. Co, Redwood City, 1990.

[10] D. H. Lyth and A. Riotto, Phys.Rept. 314 (1999) 1-146.

[11] A. Riotto and M. Trodden, Ann. Rev. Nucl. Part. Sci. 49, 35 (1999); M. Trodden, Rev.Mod.Phys. 71, 1463 (1999).

[12] D. Boyanovsky, H. J. de Vega, R. Holman, Erice Lectures on Inflationary Reheating, in the Proceedings of the 5th. Erice Chalonge School on Astrofundamental Physics, N. Sánchez and A. Zichichi eds., World Scientific, 1997. D. Boyanovsky, H. J. de Vega and R. Holman, in Proceedings of the Second Paris Cosmology Colloquium, Observatoire de Paris, 1994, edited by H. J. de Vega and N. Sánchez, World Scientific, Singapore 1995, pp. 127-215; in Advances in Astrofundamental Physics, Erice Chalonge School, edited by N. Sánchez and A. Zichichi, World Scientific, Singapore 1995; D. Boyanovsky, H. J. de Vega, R. Holman, D.-S. Lee and A. Singh, Phys. Rev. D 51, 4419 (1995); D. Boyanovsky, H. J. de Vega, R. Holman and J. Salgado, Phys. Rev. D 54, 7570 (1996); D. Boyanovsky, H. J. de Vega and R. Holman, in Current Topics in Astrofundamental Physics, Vth Erice Chalonge School, edited by N. Sánchez and A. Zichichi, World Scientific, Singapore 1996, pp. 183-270; D. Boyanovsky, H. J. de Vega, R. Holman and D.-S. Lee, Phys. Rev. D 52, 6805 (1995); D. Boyanovsky, M. D'Attanasio, H. J. de Vega and R. Holman, Phys. Rev. D 54, 1748 (1996).

[13] A. Shnirman and G. Schön, cond-mat/0210023 D. S. Golubev, G. Schön and A. D. Zaikin, cond-mat/0208548

[14] H. Haug and A. P. Jauho, Quantum Kinetics in Transport and Optics of Semiconductors, Springer, New York, 1998; J. Shah, Ultrafast Spectroscopy of Semiconductors and Semiconductor Nanostructures, Springer, New York, 1999.

[15] L. Bányai, D. B. Tran Thoai, E. Reitsamer, H. Haug, D. Steinbach, M. U. Wehner, M. Wegener, T. Marschner, and W. Stolz, Phys. Rev. Lett. 75, 2188 (1995); S. Bar-Ad, P. Kner, M. V. Marquezini, D. S. Chemla, and K. El Sayed, 77, 3177 (1996); C. Fürst, A. Leitenstorfer, A. Laubereau, and R. Zimmermann, 78, 3733 (1997).

[16] R. D. Pisarski, Phys. Rev. Lett. 63, 1129 (1989); Phys. Rev. D 47, 5589 (1993).

[17] J.-P Blaizot and E. Iancu, Phys. Rev. Lett. 76, 3080 (1996); Phys. Rev. D 55, 973 (1997); Phys. Rev. D 56, 7877 (1997). K. Takashiba, Int. J. Mod. Phys. A 11, 2309 (1996).

[18] D. Boyanovsky, H. J. de Vega, R. Holman and M. Simionato, Phys. Rev. D 60, 065003 (1999). 
[19] S. - Y. Wang, D. Boyanovsky, H. J. de Vega and D.-S. Lee, Phys. Rev. D 62, 105026 (2000); D. Boyanovsky, H. J. de Vega, S.-Y. Wang, Phys.Rev. D61 (2000) 065006; S.-Y. Wang, hep-ph/0110220 Ph.D. Thesis.

[20] L.-Y. Chen, N. Goldenfeld and Y. Oono, Phys. Rev. Lett. 73, 1311 (1994); Phys. Rev. E 54, 376 (1996).

[21] T. Kunihiro, Prog. Theor. Phys. 94, 503 (1995), ibid 97, 179 (1997), Japan J. Indus. Appl. Math. 14, 51 (1997); T. Kunihiro, Prog. Theor. Phys. 97, 179 (1997); T. Kunihiro, Prog. Theor. Phys. Suppl. 131, 459 (1998); S.-I. Ei, K. Fujii and T. Kunihiro, Ann. of Phys. (NY) 280, 236 (2000); T. Kunihiro, Phys.Rev. D57 2035 (1998); Y. Hatta and T. Kunihiro, Annals Phys. 298, 24 (2002).

[22] H. J. de Vega and J.F.J. Salgado, Phys. Rev. D 56 6524, (1997); M. Frasca, Phys. Rev. A 56, 1549 (1997); I. L. Egusquiza and M. A. Valle Basagoiti, Phys. Rev. D57, 1586 (1998); C. M. Bender and L. M. A. Bettencourt, Phys. Rev. Lett. 77, 4114 (1996), Phys. Rev. D54, 7710 (1996). J. F. J. Salgado, Thèse de Doctorat, Université de Paris VI, 1999; C. M. Bender and L. M. A. Bettencourt, Phys. Rev. Lett. 77, 4114 (1996), Phys. Rev. D54, 7710 (1996).

[23] D. Boyanovsky, H. J. de Vega and S.-Y. Wang, hep-ph/0212107

[24] J. Schwinger, J. Math. Phys. 2, 407 (1961); K. T. Mahanthappa, Phys. Rev. 126, 329 (1962); P. M. Bakshi and K. T. Mahanthappa, J. Math. Phys. 41, 12 (1963); L. V. Keldysh, JETP 20, 1018 (1965); K. Chou, Z. Su, B. Hao And L. Yu, Phys. Rep. 118, 1 (1985); A. Niemi and G. Semenoff, Ann. of Phys. (NY) 152, 105 (1984); N. P. Landsmann and C. G. van Weert, Phys. Rep. 145, 141 (1987); E. Calzetta and B. L. Hu, Phys. Rev. D41, 495 (1990); ibid D37, 2838 (1990); J. P. Paz, Phys. Rev. D41, 1054 (1990); ibid D42, 529(1990).

[25] D. Boyanovsky, H. J. de Vega and R. Holman, Proceedings of the Second Paris Cosmology Colloquium, Observatoire de Paris, June 1994, pp. 127-215, H. J. de Vega and N. Sánchez, Editors, World Scientific, 1995; Advances in Astrofundamental Physics, Erice Chalonge School, N. Sánchez and A. Zichichi Editors, World Scientific, 1995; D. Boyanovsky, H. J. de Vega, R. Holman, D.-S. Lee and A. Singh, Phys. Rev. D51, 4419 (1995); D. Boyanovsky, H. J. de Vega, R. Holman and J. Salgado, Phys. Rev. D54, 7570 (1996).

[26] D. Boyanovsky, H. J. de Vega and R. Holman, Vth. Erice Chalonge School, Current Topics in Astrofundamental Physics, N. Sánchez and A. Zichichi Editors, World Scientific, 1996, p. 183-270. D. Boyanovsky, H. J. de Vega, R. Holman and D.-S. Lee, Phys. Rev. D52, 6805 (1995).

[27] D. Boyanovsky, M. D'Attanasio, H. J. de Vega, R. Holman and D. S. Lee, Phys. Rev. D52, 6805 (1995). D. Boyanovsky, M. D'Attanasio, H. J. de Vega and R. Holman, Phys. Rev. D54, 1748 (1996).

[28] S. Weinberg, The Quantum Theory of Fields I, Cambridge University Press, 1995 and references therein.

[29] G. D. Mahan, Many-Particle Physics, 2nd Edition, Plenum Press, N.Y. 1990, see chapter 8; S. Doniach and E. H. Sondheimer, Green's functions for Solid State Physicists (Imperial College Press, Singapore, 1999) (see chapter 9).

[30] D. Boyanovsky and H. J. de Vega, Phys.Rev. D63, 034016 (2001).

[31] J. I. Kapusta, Finite Temperature Field Theory, Cambridge University Press, 1989.

[32] M. le Bellac, Thermal Field Theory, Cambridge University Press, 1996.

[33] See for example: G. Baym, Lectures on Quantum Mechanics W. A. Benjamin, Inc, N.Y., 1969. 